\title{
INSECURITY FOR COMPACT SURFACES OF POSITIVE GENUS
}

\author{
VICTOR BANGERT AND EUGENE GUTKIN
}

\section{Contents}

1. Introduction

2. Rays, corays, and Busemann functions

3. Outline of the proof that nonflat two-tori are insecure 6

4. Minimal geodesics in an admissible strip 9

5. The key lemma 10

6. Nonflat two-dimensional tori are insecure 16

7. Blocking and insecurity for tori of revolution 18

8. Insecurity for all point pairs in a two-dimensional torus 21

9. Total insecurity for surfaces of genus greater than one 29

References 36

\begin{abstract}
A pair of points in a riemannian manifold $M$ is secure if the geodesics between the points can be blocked by a finite number of point obstacles; otherwise the pair of points is insecure. A manifold is secure if all pairs of points in $M$ are secure. A manifold is insecure if there exists an insecure point pair, and totally insecure if all point pairs are insecure.

Compact, flat manifolds are secure. A standing conjecture says that these are the only secure, compact riemannian manifolds. We prove this for surfaces of genus greater than zero. We also prove that a closed surface of genus greater than one with any riemannian metric and a closed surface of genus one with generic metric are totally insecure.
\end{abstract}

Date: March 5, 2022.

1991 Mathematics Subject Classification. 53C22,57M10,37E40.

Key words and phrases. compact riemannian surface, minimal geodesic, closed geodesic, free homotopy class, blocking set, insecurity, flat torus, surface of genus greater than one. 


\section{InTRODUCTION}

We begin by describing our setting and establishing the terminology. By a riemannian manifold $(M, g)$ we will mean a complete, connected, infinitely differentiable riemannian manifold. We will view geodesics in $(M, g)$ as curves, $c: I \rightarrow M$, parameterized by arclength, where $I \subset \mathbb{R}$ is any nontrivial interval. The set $c(I) \subset M$ is the trace of $c$. If $I=[a, b]$, we will also say that $c(I)$ is a geodesic segment; the points $x=c(a), y=c(b)$ are the endpoints of the geodesic. If $z \in M$ is an interior point of $c(I)$, we say that $c$ passes through $z$.

For any pair $x, y \in M$ (including $y=x$ ) let $G(x, y)$ be the set of geodesic segments in $(M, g)$ with endpoints $x, y$. We say that these geodesics join $x$ with $y$. A finite set $B \subset M \backslash\{x, y\}$ is a blocking set for the pair $x, y$ if every geodesic in $G(x, y)$ passes through a point $b \in B$. We will also say that $B$ blocks $x$ and $y$ away from each other.

A pair $x, y \in M$ is insecure if these points cannot be blocked away from each other. Otherwise, the pair $(x, y)$ is secure. A riemannian manifold is insecure if it contains an insecure pair of points. Thus, $(M, g)$ is secure if any point in it can be blocked away from any point, including itself. Finally, $(M, g)$ is totally insecure if every pair $x, y \in M$ is insecure. See Gut05] for a motivation of this terminology.

Which compact riemannian manifolds are secure? The only examples so far are the flat manifolds GS06. Researchers in the subject believe in the following statement BG08, LS07].

Conjecture 1. A compact riemannian manifold is secure if and only if it is flat.

Restricting the setting, we state a counterpart of Conjecture 1 for tori.

Conjecture 2. A riemannian torus is secure if and only if it is flat.

In this note we obtain several results concerning security of closed riemannian surfaces. In particular, we establish Conjecture 1 for twodimensional tori. See Theorem 6.4.

Theorem 1. A two-dimensional riemannian torus is secure if and only if it is flat.

Security and insecurity are preserved under finite coverings [GS06]. This observation allows us to restrict the discussion to orientable manifolds. Our next result concerns the insecurity of closed surfaces of genus greater than one. See Theorem 9.7 
Theorem 2. A closed riemannian surface of genus greater than one is totally insecure.

Since surfaces of genus greater than one do not admit flat riemannian metrics, this Theorem provides evidence for Conjecture 1 .

An insecure manifold, in particular, a nonflat two-torus, may have secure pairs of points. In section 7 we analyze the security of point pairs on two-dimensional tori of revolution. This class of riemannian tori contains the round euclidean tori of revolution $T^{2} \subset \mathbb{R}^{3}$. Let $E_{\text {in }} \subset T^{2}$ be the inner equator. Our Corollary 7.3 says the following.

Proposition 1. Let $T^{2} \subset \mathbb{R}^{3}$ be a round euclidean torus of revolution. Let $x, y \in T^{2}$ be any pair of points. Then it is secure if and only if $x, y \in E_{\text {in }}$.

Tori of revolution are special. By our Corollary 8.7, a generic riemannian two-torus is totally insecure. Here is the precise statement.

Corollary 1. The set of riemannian two-tori contains a $C^{2}$-open and $C^{\infty}$-dense subset of totally insecure tori.

Conjecture 1 holds for locally symmetric spaces [GS06]. Moreover, compact locally symmetric spaces of noncompact type are totally insecure GS06. This is true, in particular, for compact surfaces of constant negative curvature. A geometric argument showing this is sketched in GS06. Let $M$ be a surface as above, let $x, y \in M$ be a pair of points, and let $C \subset M \backslash\{x, y\}$ be a periodic geodesic. The argument in question constructs an infinite sequence of geodesics $\gamma_{n} \in G(x, y)$. As $n \rightarrow \infty$, the geodesics $\gamma_{n}$, their lengths going to infinity, spend almost all their time in ever smaller vicinity of $C$, never intersecting it. Thus, through any point $z \in M \backslash\{x, y\}$ passes at most a finite number of geodesics $\gamma_{n}$.

Our proofs of Theorem 1 and Theorem 2 use a similar idea, although the two approaches differ considerably in detail. Since we are working with arbitrary riemannian metrics, we cannot use the hyperbolicity of the geodesic flow, which was crucial for the analysis in GS06. Instead, we use the classical results of Morse [Mo] and Hedlund [He], as well as more recent results [Ba1], [Ba2], [In], on minimal geodesics in surfaces to construct infinite sequences of joining geodesics with a similar behavior. See section 4 and section 5. Our Proposition 6.1 says that once we have an infinite sequence of geodesics joining points $x, y$ 
and satisfying certain conditions, the pair $(x, y)$ is insecure. The strategy of proving Theorem 1 and Theorem 2 is to construct in each case sequences of geodesics satisfying the requirements of Proposition 6.1.

Another approach to insecurity for riemannian manifolds is based on connections between the insecurity and the growth rate of the number of joining geodesics. See [BG08], [LS07, and Gut09]. This approach works well under additional assumptions, e. g., that the manifold $(M, g)$ has no conjugate points. Then we can use the well known relationships between the growth rate of the number $n_{T}(x, y)$ of joining geodesics having length $\leq T$ and the growth of $\pi_{1}(M)$, as well as those between $n_{T}(x, y)$ and the topological entropy of $(M, g)$.

Acknowledgements. The work of $\mathrm{V}$. Bangert was partially supported by SFB/Transregio 71 "Geometrische Partielle Differentialgleichungen". E. Gutkin did some of the work while visiting FIM at ETH, Zurich, the Department of Mathematics at UCLA, and the Mathematisches Institut at Albert-Ludwigs-Universität in Freiburg im Breisgau. It is a pleasure to thank these institutions for hospitality and financial support.

\section{RAYS, CORAYS, AND BUSEMANn FUnCTIONS}

In this section we review basic material on rays, corays and Busemann functions in riemannian manifolds. We will use the notation $\left(M^{n}, g\right)$ for riemannian manifolds, suppressing $g$ and/or $n$ whenever this causes no confusion. We denote by $d(\cdot, \cdot)$ the riemannian distance on $M$. We will view geodesics as parameterized curves $c(t), t \in I$, where $I \subset \mathbb{R}$ is a nontrivial, possibly infinite interval, and $t$ is an arclength parameter. We will call the set $c(I) \subset M$ the trace of $c$.

Definition 2.1. Let $I \subset \mathbb{R}$ be an interval and let $c: I \rightarrow M$ be a geodesic.

(a) The geodesic $c$ is minimal if $d(c(t), c(s))=|t-s|$ for all $s, t \in I$.

(b) A ray is a minimal geodesic $c: \mathbb{R}_{\geq 0} \rightarrow M$.

(c) Let $c: \mathbb{R}_{\geq 0} \rightarrow M$ be a ray, and let $C \subset M$ be its trace. $A$ ray $\tilde{c}$ is a coray to $c$ if there exists a sequence of minimal geodesics $c_{n}:\left[0, L_{n}\right] \rightarrow M$ with $\lim _{n \rightarrow \infty} L_{n}=\infty$, such that $\lim _{n \rightarrow \infty} \dot{c}_{n}(0)=\dot{\tilde{c}}(0)$ and $c_{n}\left(L_{n}\right) \in C$ for all $n \in \mathbb{N}$.

Throughout the paper we will denote by $L(\gamma)$ the length of a curve.

Definition 2.2. A geodesic $c: I \rightarrow M$ is homotopically minimal if its lifts to the universal riemannian covering of $M$ are minimal. 
Equivalently, a geodesic $c: I \rightarrow M$ is homotopically minimal iff the following holds. Let $s, t \in I$ and let $s<t$. Let $\gamma:[s, t] \rightarrow M$ be a curve satisfying $\gamma(s)=c(s), \gamma(t)=c(t)$ and such that the curves $\gamma$ and $\left.c\right|_{[s, t]}$ are homotopic with fixed endpoints. Then

$$
L(c([s, t])) \leq L(\gamma)
$$

Throughout the paper we will use the following basic result. It is essentially due to M. Morse. See [Mo, Theorems 9 and 10.

Theorem 2.3. Let $M$ be an orientable surface, and let $c: \mathbb{R} \rightarrow M$ be a periodic geodesic with period $L>0$. If the closed curve $\left.c\right|_{[0, L]}$ has minimal length among all closed curves freely homotopic to $\left.c\right|_{[0, L]}$, then $c$ is homotopically minimal.

Taking limits of minimal geodesics of finite length, we obtain the following basic fact.

Proposition 2.4. A complete riemannian manifold $(M, g)$ carries a ray if and only if it is not compact. If $c$ is a ray in $(M, g)$ and $p \in M$, then there exists a coray $\tilde{c}$ to $c$ with $\tilde{c}(0)=p$.

Definition 2.5. Let $c: \mathbb{R}_{+} \rightarrow M$ be a ray. Its Busemann function, $B_{c}: M \rightarrow \mathbb{R}$, is defined by

$$
B_{c}(p)=\lim _{t \rightarrow \infty}[d(p, c(t))-t]
$$

By the triangle inequality, the function $t \rightarrow d(p, c(t))-t$ is monotonically decreasing 1 Also by the triangle inequality, it satisfies $-d(c(0), p) \leq$ $d(p, c(t))-t$. Thus, the limit in equation (1) exists.

Lemma 2.6. Let $c: \mathbb{R}_{+} \rightarrow M$ be a ray, and let $p, q \in M$ be arbitrary points. Then

$$
\left|B_{c}(p)-B_{c}(q)\right| \leq d(p, q) .
$$

Proof. Apply the triangle inequality to the triangle with corners $p, q, c(t)$, and take the limit $t \rightarrow \infty$.

By Lemma 2.6, any Busemann function is lipschitz, with the lipschitz constant 1.

\footnotetext{
${ }^{1}$ In general, not strictly.
} 
Proposition 2.7. Let $c: \mathbb{R}_{+} \rightarrow M$ be a ray. A geodesic $\tilde{c}: \mathbb{R}_{+} \rightarrow M$ is a coray to $c$ if and only if for all $s, t \in \mathbb{R}_{+}$the equation

$$
B_{c}(\tilde{c}(t))-B_{c}(\tilde{c}(s))=s-t
$$

holds.

Proof. This follows from equations (22.16) and (22.20) in $\mathrm{Bu}$.

We use Proposition 2.7 to relax the requirements in Definition 2.1.

Lemma 2.8. Let $c: \mathbb{R}_{+} \rightarrow M$ be a ray, and let $C \subset M$ be its trace. Let $L_{n} \rightarrow \infty$ be a positive sequence. Let $c_{n}:\left[0, L_{n}\right] \rightarrow M$ be minimal geodesics such that $\lim _{n \rightarrow \infty} d\left(c_{n}\left(L_{n}\right), C\right)=0$.

If $\tilde{c}: \mathbb{R}_{+} \rightarrow M$ is a geodesic such that $\dot{\tilde{c}}(0)$ is a point of accumulation of the sequence $\dot{c}_{n}(0)$, then $\tilde{c}$ is a coray to $c$.

Proof. We assume without loss of generality that $\lim _{n \rightarrow \infty} \dot{c}_{n}(0)=\dot{\tilde{c}}(0)$. For every $n \in \mathbb{N}$ there is a number $t_{n} \in[0, \infty)$ such that the sequence $\varepsilon_{n}=d\left(c_{n}\left(L_{n}\right), c\left(t_{n}\right)\right)$ converges to zero. Since the geodesics $c_{n}$ are minimal, the condition $\lim _{n \rightarrow \infty} L_{n}=\infty$ implies that $\lim _{n \rightarrow \infty} t_{n}=\infty$. By the triangle inequality, for all $n$ and any $t \in\left[0, L_{n}\right]$ we have

$$
L_{n}-t-\varepsilon_{n} \leq d\left(c_{n}(t), c\left(t_{n}\right)\right) \leq L_{n}-t+\varepsilon_{n} .
$$

Let $s, t>0$ be arbitrary. Using that $\lim \dot{c}_{n}(0)=\dot{\tilde{c}}(0)$ and equation (2), we have

$$
\begin{array}{r}
B_{c}(\tilde{c}(t))-B_{c}(\tilde{c}(s))=\lim _{n \rightarrow \infty}\left[d\left(\tilde{c}(t), c\left(t_{n}\right)\right)-d\left(\tilde{c}(s), c\left(t_{n}\right)\right)\right] \\
=\lim _{n \rightarrow \infty}\left[d\left(c_{n}(t), c\left(t_{n}\right)\right)-d\left(c_{n}(s), c\left(t_{n}\right)\right)\right] \geq \lim _{n \rightarrow \infty}\left(s-t-2 \varepsilon_{n}\right)=s-t .
\end{array}
$$

Combining this inequality with Lemma 2.6, we obtain

$$
B_{c}(\tilde{c}(t))-B_{c}(\tilde{c}(s))=s-t .
$$

The claim now follows from Proposition 2.7.

\section{Outline of the PROOF THAT NONFlat TWO-TORI ARE INSECURE}

For the benefit of the reader, we will outline the main ideas in the proof of Theorem 6.4 which is Theorem 1 in the Introduction. The methods used in this proof are also typical for several other statements in this paper. Let $\left(T^{2}, \bar{g}\right)$ be a non-flat two-dimensional torus; our goal is to find a pair of points in $\left(T^{2}, \bar{g}\right)$ that cannot be blocked away from each other by a finite blocking set.

By a classical theorem of E. Hopf [Ho, a riemannian two-torus is flat if and only if it has no conjugate points. Thus, the torus $\left(T^{2}, \bar{g}\right)$ 
has conjugate points. Then, by a theorem of N. Innami, there exists a nontrivial free homotopy class $\alpha$ of closed curves such that $\left(T^{2}, \bar{g}\right)$ cannot be foliated by geodesics in $\alpha$. See [In], Corollary 3.2; see also the proof of Theorem 6.1 in [Ba2].

Let $\mathcal{M}_{\alpha}^{\text {per }}$ be the set of periodic geodesics of minimal length in the class $\alpha$. By results that go back to M. Morse [Mo] and G. Hedlund $[\mathrm{He}$, these geodesics do not self-intersect and are pairwise disjoint. Generically, $\mathcal{M}_{\alpha}^{\text {per }}$ consists of a single geodesic.

The geodesics in $\mathcal{M}_{\alpha}^{\text {per }}$ foliate a compact, proper subset, $N \subset T^{2}$. Let $Z \subset T^{2}$ be a connected component of $T^{2} \backslash N$; let $p, q \in Z$ be any pair of points. We will show that the pair $p, q$ is insecure, i. e., that we cannot block $p$ away from $q$ by a finite blocking set.

We denote by $\left(\mathbb{R}^{2}, g\right)$ the riemannian universal covering; let $\pi$ : $\left(\mathbb{R}^{2}, g\right) \rightarrow\left(T^{2}, \bar{g}\right)$ be the projection. Let $S \subset \mathbb{R}^{2}$ be a connected component of $\pi^{-1}(Z)$. Then $S$ is an open strip. The boundary $\partial S$ is a disjoint union of traces of two geodesics, $c_{0}: \mathbb{R} \rightarrow\left(\mathbb{R}^{2}, g\right)$ and $c_{1}: \mathbb{R} \rightarrow\left(\mathbb{R}^{2}, g\right)$. By Theorem 2.3, the geodesics $c_{0}, c_{1}$ are minimal. Let $C_{0}, C_{1}$ be the respective traces; then $\partial S=C_{0} \cup C_{1}$.

Let $P, Q_{0} \in S$ be arbitrary points such that $\pi(P)=p, \pi\left(Q_{0}\right)=q$. Using the action of the stabilizer of $S$ in $\pi_{1}\left(T^{2}\right)=\mathbb{Z}^{2}$, we produce an infinite sequence of points $Q_{1}, \ldots, Q_{n}, \ldots \in S$ such that $\pi\left(Q_{n}\right)=q$ and the sequence of distances $L_{n}=d\left(P, Q_{n}\right)$ goes to infinity. Let now $\tilde{c}_{n}:\left[0, L_{n}\right] \rightarrow S$ be a sequence of minimal geodesics such that $\tilde{c}_{n}(0)=P$ and $\tilde{c}_{n}\left(L_{n}\right)=Q_{n}$.

Lemma 5.1 in section 5 implies that most of the time the geodesics $\tilde{c}_{n}$ are close to $\partial S$. More precisely, for any $\varepsilon>0$ there exists $T=T(\varepsilon)>0$ such that for all $t \in\left[T, L_{n}-T\right]$ the points $\tilde{c}_{n}(t)$ are $\varepsilon$-close to $\partial S$. Figure 1 illustrates the behavior of this sequence of geodesics.

Set $c_{n}=\pi \circ \tilde{c}_{n}$. Then the geodesics $c_{n}:\left[0, L_{n}\right] \rightarrow Z$ join the points $p, q$. Let $z \in Z \backslash\{p, q\}$ be an arbitrary point. The preceding discussion implies that at most a finite number of the geodesics $c_{n}$ passes through $z$. On the other hand, if $z \in T^{2} \backslash Z$, no geodesic in our sequence passes through it. Thus, any point $z \in T^{2}$ can block at most a finite number of joining geodesics in the infinite sequence $c_{n}$. Hence, we cannot block the points $p, q$ away from each other by a finite blocking set.

We will now illustrate the preceding discussion with the example of round euclidean tori of revolution.

Example 3.1. Let $0<r<R$, and set $C=C(r, R)=\{(x, 0, z)$ : $\left.(x-R)^{2}+z^{2}=r^{2}\right\}$. This is a circle of radius $r$ in the $x z$-plane. The 
$C_{1}$

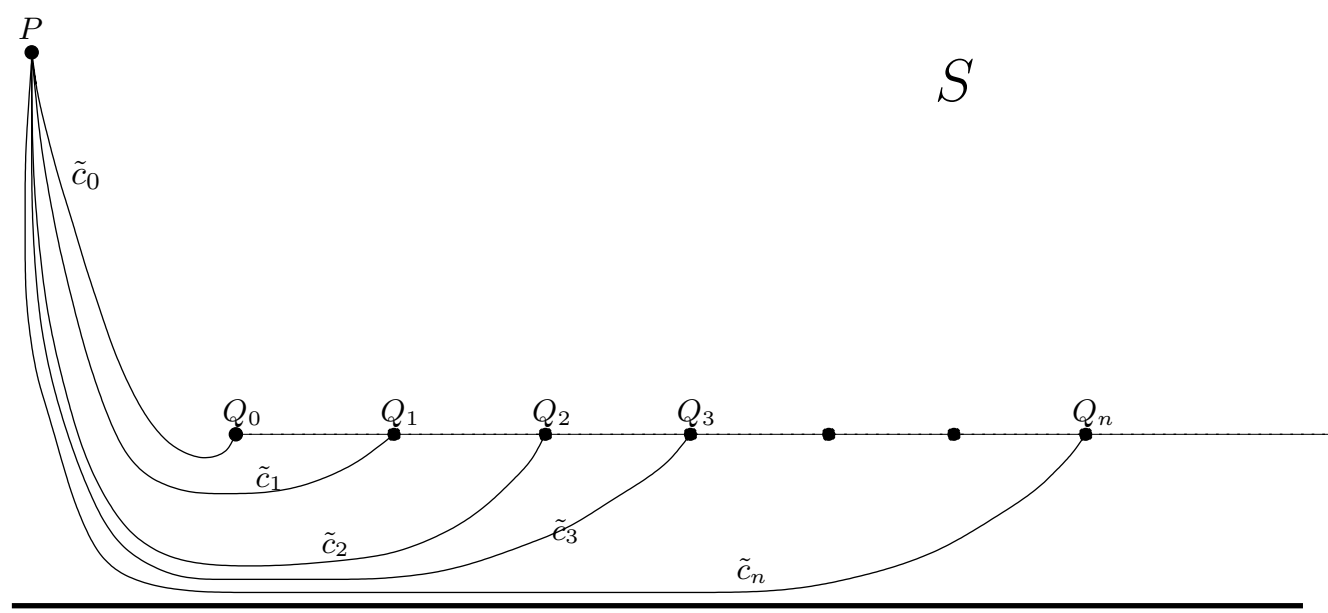

$C_{0}$

Figure 1. A sequence of minimal geodesics in the universal covering hose projections to the torus cannot be blocked by a finite point set.

round euclidean torus of revolution, $T^{2}=T^{2}(r, R) \subset \mathbb{R}^{3}$, is obtained by revolving $C$ about the $z$-axis. Points in $C$ yield the circles of latitude in $T^{2}$. Let $\alpha$ be their free homotopy class.

Let the inner equator $E_{\text {in }} \subset T^{2}$ (resp. the outer equator $E_{\text {out }} \subset$ $\left.T^{2}\right)$ be the circle of latitude generated by the point $(R-r, 0,0) \in C$ (resp. $(R+r, 0,0) \in C)$. Their lengths are $2 \pi(R-r)$ and $2 \pi(R+r)$ respectively. These are the only circles of latitude that are geodesics in the round euclidean torus.

Specializing the preceding discussion to the torus of revolution, we have $N=E_{\text {in }}$ and $Z=T^{2} \backslash E_{\text {in. }}$. The set $\mathcal{M}_{\alpha}^{\text {per }}$ consists of a single geodesic; although the tori of revolution are very special, this is the generic situation for two-dimensional riemannian tori. See section 8 . By the preceding argument, no points $p, q \in T^{2} \backslash E_{\text {in }}$ can be blocked away from each other by a finite blocking set.

In section 7 we will completely analyze the blocking for a class of two-dimensional tori containing the above tori of revolution. 


\section{Minimal GeOdesics in AN ADMissible STRIP}

We will use the notation of section 3, in particular, we use the identification $\left(T^{2}, \bar{g}\right)=\left(\mathbb{R}^{2}, g\right) / \mathbb{Z}^{2}$. If $S \subset \mathbb{R}^{2}$, we denote by

$$
\operatorname{Stab}(S)=\left\{j \in \mathbb{Z}^{2}: S+j=S\right\}
$$

the stabilizer of $S$. Recall that a nonzero vector $j \in \mathbb{Z}^{2}$ is prime if there do not exist $n \in \mathbb{N}, n \geq 2$, and $k \in \mathbb{Z}^{2}$ such that $j=n k$.

We will use the notation $\bar{X}$ for the closure of a set.

Proposition 4.1. Let $\left(T^{2}, \bar{g}\right)$ be a nonflat riemannian torus. Let $\left(\mathbb{R}^{2}, g\right)$ be its universal covering, and let $\pi:\left(\mathbb{R}^{2}, g\right) \rightarrow\left(T^{2}, \bar{g}\right)$ be the projection.

Then there exists a connected open set $S \subset \mathbb{R}^{2}$ with totally geodesic boundary, such that the following statements hold.

(a) The group $\operatorname{Stab}(S)$ is generated by a prime vector.

(b) If $j \in \mathbb{Z}^{2} \backslash \operatorname{Stab}(S)$, then $(S+j) \cap \bar{S}=\emptyset$.

(c) The boundary of $S$ has two connected components, say $C_{0}$ and $C_{1}$. There are minimal geodesics $c_{0}, c_{1}: \mathbb{R} \rightarrow\left(\mathbb{R}^{2}, g\right)$ whose traces are $C_{0}$ and $C_{1}$, respectively.

(d) Let $c: \mathbb{R} \rightarrow S$ be a geodesic such that $\pi \circ c: \mathbb{R} \rightarrow T^{2}$ is periodic. Then $c$ is not minimal.

Proof. By Corollary 3.2 in [In], there exists a nontrivial free homotopy class, say $\alpha$, of closed curves in $T^{2}$ having the following property: There does not exist a family of closed geodesics in the class $\alpha$ whose traces foliate $T^{2}$. We can assume that $\alpha$ is prime.

Let $L$ be the minimal length of a curve in $\alpha$; we denote by $\mathcal{M}_{\alpha}^{\text {per }}$ the set of closed geodesics in the class $\alpha$ having length $L$. Clearly, $\mathcal{M}_{\alpha}^{\text {per }} \neq \emptyset$. By Theorem 6.5 and Theorem 6.6 in [Ba1], the trace of every $c \in \mathcal{M}_{\alpha}^{\text {per }}$ is an embedded curve in $T^{2}$. Moreover, if $c, \tilde{c} \in \mathcal{M}_{\alpha}^{\text {per }}$, then either their traces are disjoint or $c$ and $\tilde{c}$ coincide up to a translation of the parameter.

Let $N$ be the union of the traces of geodesics in $\mathcal{M}_{\alpha}^{\text {per }}$. By our choice of $\alpha$, the set $N \subset T^{2}$ is a proper, nonempty, closed subset. Let $Z$ be a connected component of $T^{2} \backslash N$. Let $\partial Z=\bar{Z} \backslash Z$ be its boundary. Then either $\partial Z$ is the trace of a geodesic in $\mathcal{M}_{\alpha}^{\text {per }}$ or $\partial Z$ is the union of traces of two geodesics in $\mathcal{M}_{\alpha}^{\text {per }} 2$

Let $S$ be a connected component of $\pi^{-1}(Z) \subset \mathbb{R}^{2}$. Then the boundary of $S$ is the union of the traces of two geodesics $c_{0}, c_{1}: \mathbb{R} \rightarrow \mathbb{R}^{2}$ such

\footnotetext{
${ }^{2}$ We point out that $Z$ is homeomorphic to the cylinder $S^{1} \times(0,1)$.
} 
that $\pi \circ c_{0}$ and $\pi \circ c_{1}$ belong to $\mathcal{M}_{\alpha}^{\text {per }}$. Let $k \in \mathbb{Z}^{2}$ correspond to $\alpha$. Then for all $t \in \mathbb{R}$ we have

$$
c_{0}(t+L)=c_{0}(t)+k, c_{1}(t+L)=c_{1}(t)+k .
$$

Theorem 2.3 implies that $c_{0}$ and $c_{1}$ are homotopically minimal geodesics. The remaining statements in (a), (b), and (c) now follow by elementary topological arguments; claim (d) follows from Theorem 6.7 in [Ba1].

In what follows an open set $S \subseteq \mathbb{R}^{2}$ satisfying the conditions stated in Proposition 4.1 will be called an admissible strip; the projection $Z=\pi(S) \subseteq T^{2}$ is an admissible cylinder.

\section{THE KEY LEMMA}

We will use the setting and the notation of section 4. In particular, $S$ will denote an admissible strip. The following statement is crucial in our proof of Theorem 6.4. We will refer to it as the Key Lemma.

Lemma 5.1. For any $\varepsilon>0$ there exists $T=T(\varepsilon)>0$ such that the following holds. If $c:[0, L] \rightarrow S$ is a minimal geodesic and $d(c(0), \partial S) \geq \varepsilon$ then $d(c(t), \partial S) \leq \varepsilon$ for all $t \in[T, L-T]$.

The proof of Lemma 5.1 is based on the results of M. Morse [Mo] about minimal geodesics in $S$ and on a result from Ba2 concerning the rays in $S$. We need a few technical lemmas.

Lemma 5.2. Let $c:[0, \infty) \rightarrow S$ be a ray. Then $\lim _{t \rightarrow \infty} d(c(t), \partial S)=$ 0 .

Proof. The claim follows from Theorem 3.7 in [Ba2], interpreted as a statement about minimal geodesics in $\left(\mathbb{R}^{2}, g\right)$. See Example (1) on page 51 in [Ba2] for details.

Throughout this section we will use the following notational conventions. With any geodesic $c: \mathbb{R} \rightarrow \bar{S}$ we will associate two geodesics $c_{ \pm}: \mathbb{R}_{+} \rightarrow \bar{S}$ as follows. The geodesic $c_{+}$is the restriction of $c$ to the positive half-line. We define the geodesic $c_{-}$by $c_{-}(t)=c(-t)$.

We will denote by $C, C_{+}, C_{-} \subset \bar{S}$ the respective traces of $c, c_{+}, c_{-}$.

Definition 5.3. Let $c_{0}, c_{1}: \mathbb{R} \rightarrow \bar{S}$ be two geodesics such that their traces $C_{0}, C_{1}$ are the two components of $\partial S$. We say that the geodesics $c_{0}, c_{1}$ are coherently oriented if for any time sequence $t_{n} \rightarrow \infty$ the two point sequences $c_{0}\left(t_{n}\right), c_{1}\left(t_{n}\right) \in \partial S$ converge to the same end of $\bar{S}$. 
$C_{1}$

\section{$C_{0}$}

FiguRE 2. A strip with coherently oriented boundary components.

Figure 2 illustrates Definition 5.3. We will also say that $c_{0}, c_{1}: \mathbb{R} \rightarrow$ $\bar{S}$ are coherent parameterizations of $\partial S$.

Lemma 5.4. Let $\partial S=C_{0} \cup C_{1}$, where $c_{0}, c_{1}: \mathbb{R} \rightarrow \bar{S}$ are coherently oriented. Let the geodesics $c_{0, \pm}: \mathbb{R}_{+} \rightarrow \partial S$ and $c_{1, \pm}: \mathbb{R}_{+} \rightarrow \partial S$ be as above; let $C_{0, \pm} \subset \partial S$ and $C_{1, \pm} \subset \partial S$ be the respective traces.

Let now $c: \mathbb{R} \rightarrow S$ be a minimal geodesic. Then, switching $c_{0}$ with $c_{1}$ and reversing the orientation of $c$, if need be, we have

$$
\lim _{t \rightarrow-\infty} d\left(c(t), C_{0,-}\right)=0, \lim _{t \rightarrow+\infty} d\left(c(t), C_{1,+}\right)=0 .
$$

Proof. By Lemma 5.2, $c(t)$ converges to $\partial S$ as $|t| \rightarrow \infty$. By Theorem 15 in [Mo or by Theorem 6.7 in [Ba1, the equation $\lim _{t \rightarrow-\infty} d\left(c(t), C_{0,-}\right)=$ 0 implies $\lim _{t \rightarrow+\infty} d\left(c(t), C_{1,+}\right)=0$.

Remark 5.5. Let $M$ be a riemannian manifold. If $c: I \rightarrow M$ is a geodesic, its inverse is the geodesic $c^{-1}:-I \rightarrow M$ defined by $c^{-1}(t)=$ $c(-t)$. Lemma 5.4 is equivalent to the following geometric fact.

Let $Z \subset T^{2}$ be an admissible cylinder. Assume, for simplicity of exposition, that the closure of $Z$ is a proper subset of $T^{2}$. Let $\bar{c}_{0}, \bar{c}_{1}$ : $\mathbb{R} \rightarrow\left(T^{2}, \bar{g}\right)$ be the periodic geodesics in the homotopy class $\alpha$ whose respective traces are the two components of the boundary $\partial Z$.

Let now $c: \mathbb{R} \rightarrow Z$ be a geodesic whose lift $\tilde{c}: \mathbb{R} \rightarrow S$ is minimal. Then $c$ is a heteroclinic connection either between $\bar{c}_{0}$ and $\bar{c}_{1}$ or between $\bar{c}_{0}^{-1}$ and $\bar{c}_{1}^{-1}$. 
Our next lemma says that if a ray in $S$ is a coray to a ray in a boundary component of $S$, then it is asymptotic to this component.

Lemma 5.6. Let $c_{0}: \mathbb{R} \rightarrow \bar{S}$ be a geodesic whose trace is one of the components of $\partial S$.

Let $c: \mathbb{R}_{+} \rightarrow S$ be a coray to $c_{0,+}$. Then $\lim _{t \rightarrow \infty} d\left(c(t), C_{0,+}\right)=0$.

Proof. By Theorem 3.7 in Ba2, for any $q \in S$ there exists a ray $\tilde{c}: \mathbb{R}_{+} \rightarrow S$ such that $\tilde{c}(0)=q$ and $\lim _{t \rightarrow \infty} d\left(\tilde{c}(t), C_{0,+}\right)=0$. Hence, by Lemma 5.2 and Lemma 2.8, $\tilde{c}$ is a coray to $c_{0,+}$.

Set $q=c(1)$, and let $\tilde{c}: \mathbb{R}_{+} \rightarrow S$ be as above. Thus, both $c$ and $\tilde{c}$ are corays to $c_{0,+}$; by construction, $\tilde{c}(0)=c(1)$. The geodesic $t \mapsto c(1+t)$ is also a coray to $c_{0,+}$ starting at $q=c(1)=\tilde{c}(0)$. By Theorem 22.19 in [Bu] or, by Corollary 3.8 in [Ba2], there is only one coray to $c_{0,+}$ starting at $q$. Figure 3 shows a hypothetical configuration of the rays $c$ and $\tilde{c}$ which cannot materialize.

Therefore, the ray $\tilde{c}$ satisfies $\tilde{c}(t)=c(1+t)$. Since $\tilde{c}$ is asymptotic to $C_{0,+}$, the claim follows.

$C_{1}$

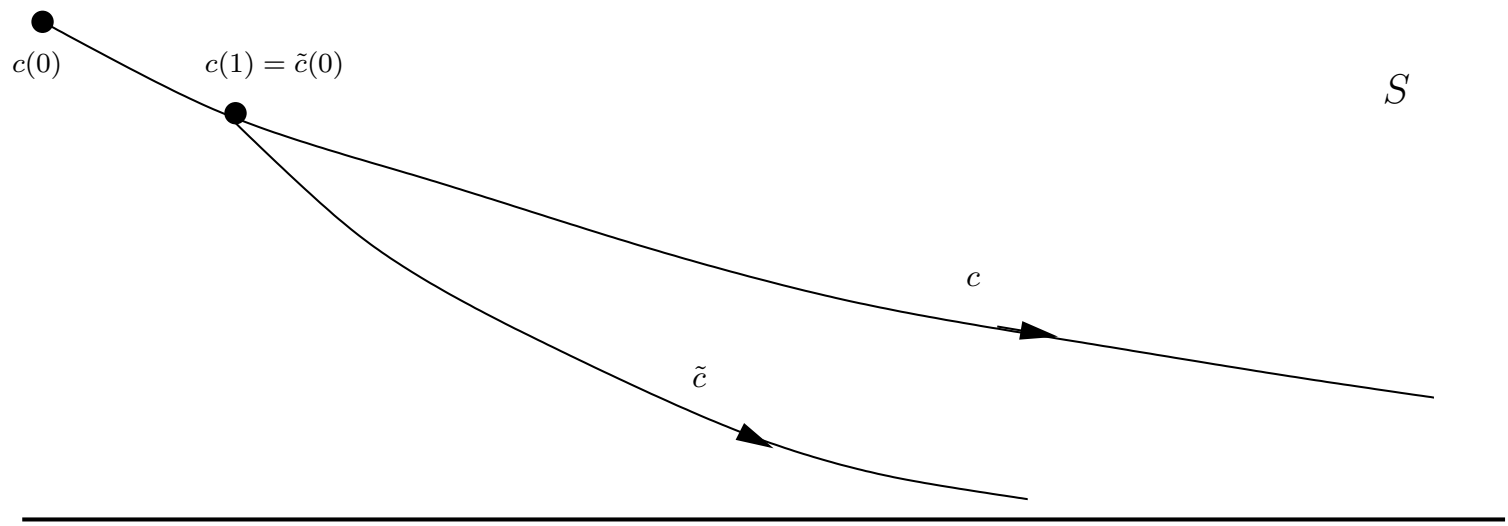

$C_{0}$

Figure 3. Illustration to the proof of Lemma 5.6. A configuration that does not exist.

We will now prove a preliminary variant of the Key Lemma.

Lemma 5.7. For any $\varepsilon>0$ there exists $\eta=\eta(\varepsilon)>0$ such that the following holds. Let $0<L<\infty$, and let $c:[0, L] \rightarrow S$ be a 
minimal geodesic such that $d(c(0), \partial S) \geq \varepsilon$ and $d\left(c(L), C_{1}\right)<\eta$. Then $d\left(c(t), C_{0}\right) \geq \eta$ for all $t \in[0, L]$.

Proof. Suppose that the claim fails. Then there exists $\delta>0$, a sequence of minimal geodesics $c_{n}:\left[0, L_{n}\right] \rightarrow S$, and a sequence $t_{n} \in$ $\left[0, L_{n}\right]$ such that $d\left(c_{n}(0), \partial S\right) \geq \delta, \lim _{n \rightarrow \infty} d\left(c_{n}\left(L_{n}\right), C_{1}\right)=0$, and $\lim _{n \rightarrow \infty} d\left(c_{n}\left(t_{n}\right), C_{0}\right)=0$.

The closed strip $\bar{S}$ is invariant under the group $\operatorname{Stab}(S) \simeq k \mathbb{Z}$ that acts on $\bar{S}$ by isometries. We have denoted this action by $z \mapsto z+r k$. We will use the same notation for the corresponding action of $\operatorname{Stab}(S)$ on geodesics in $S$. Then for any integers $r_{1}, \ldots, r_{n}, \ldots \in \mathbb{Z}$ the sequence of geodesics $\tilde{c}_{n}=c_{n}+r_{n} k$ satisfies the above conditions. In view of this observation, and the compactness of the quotient $\bar{S} / k \mathbb{Z}$, we assume without loss of generality that the vectors $\dot{c}_{n}(0)$ converge to a limit vector, $v \in T^{1}(S, g)$; let $p \in S$ be its footpoint.

We will now prove that $\lim _{n \rightarrow \infty} t_{n}=\infty$. If this fails, then, by passing to a subsequence, if need be, we have $\lim t_{n}=\bar{t}<\infty$. Let $\tilde{c}: \mathbb{R} \rightarrow \mathbb{R}^{2}$ be the geodesic with the initial vector $v$. Then $\tilde{c}(0)=p \in S$, and $\tilde{c}(\bar{t})=q=\lim _{n \rightarrow \infty} c_{n}\left(t_{n}\right) \in C_{0} \subset \partial S$. Since $\partial S$ is geodesic, $\tilde{c}$ intersects it transversally at $q$. Thus, for $t>\bar{t}$ and sufficiently close to $\bar{t}$, we have $\tilde{c}(t) \notin \bar{S}$. Figure 4 illustrates the analysis.

On the other hand, $\lim \inf L_{n} \geq \bar{t}+d\left(C_{0}, C_{1}\right)$ implies that $\tilde{c}(t) \in \bar{S}$ for all $t \in\left[0, \bar{t}+d\left(C_{0}, C_{1}\right)\right]$. In view of this contradiction, $\lim t_{n}=\infty$.

Since $\lim d\left(c_{n}\left(t_{n}\right), C_{0}\right)=0$ and $\lim t_{n}=\infty$, by Lemma 2.8, $\tilde{c}$ is a coray to $c_{0,+}$ or $c_{0,-}$. Similarly, $\lim d\left(c_{n}\left(L_{n}\right), C_{1}\right)=0$ and $\lim L_{n}=\infty$ imply, by Lemma 2.8 , that $\tilde{c}$ is a coray to $c_{1,+}$ or $c_{1,-}$. In view of Lemma 5.6, this is impossible.

We will now prove the Key Lemma. Recall that we view geodesics in $\bar{S}$ as mappings $c: I \rightarrow \bar{S}$ of nontrivial intervals $I \subset \mathbb{R}$. For $t \in I$ the velocity vectors $\dot{c}(t)$ are unit tangent vectors in $\left(\mathbb{R}^{2}, g\right)$. Thus, length $(c)=|I|$. If $0 \in I$, we will refer to $\dot{c}(0)$ as the initial vector of $c$.

Proof. (of Lemma 5.1) Suppose the claim fails. Then for some $\varepsilon>0$ there exists a sequence of minimal geodesics $c_{n}:\left[0, L_{n}\right] \rightarrow S$ such that the following conditions are satisfied:

i) For all $n \in \mathbb{N}$ we have $d\left(c_{n}(0), \partial S\right) \geq \varepsilon$;

ii) For each $n$ there is $t_{n} \in\left[n, L_{n}-n\right]$ so that $d\left(c_{n}\left(t_{n}\right), \partial S\right)>\varepsilon$.

As in the proof of Lemma 5.7, we assume without loss of generality that the velocity vectors $\dot{c}_{n}\left(t_{n}\right)$ converge to a vector $v \in T^{1}(\bar{S}, g)$. Let $\tilde{c}: \mathbb{R} \rightarrow S$ be the geodesic such that $v=\dot{\tilde{c}}(0)$. Since all of $c_{n}:\left[0, L_{n}\right] \rightarrow$ 


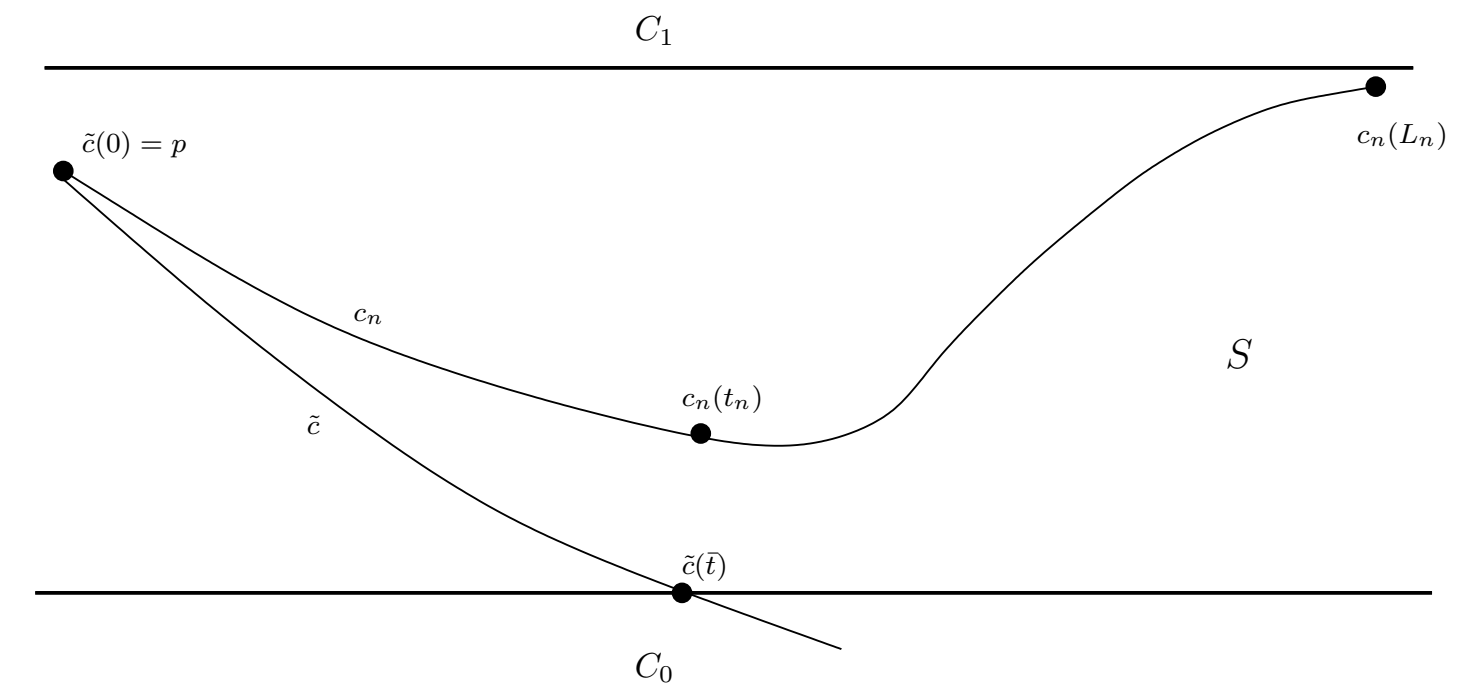

Figure 4. Illustration to the proof of Lemma 5.7; The behavior of the geodesic $\tilde{c}$ deduced from the assumption $\lim _{n \rightarrow \infty} t_{n}<\infty$.

$S$ are minimal, and $t_{n} \in\left[n, L_{n}-n\right]$, we conclude that $\tilde{c}: \mathbb{R} \rightarrow S$ is a minimal geodesic. By construction, it satisfies $d(\tilde{c}(0), \partial S) \geq \varepsilon$.

Let $\eta=\eta(\varepsilon)>0$ be as in Lemma 5.7. By Lemma 5.4, there are $s_{0}, s_{1} \in \mathbb{R}$ such that $d\left(\tilde{c}\left(s_{0}\right), C_{0}\right)<\eta$ and $d\left(\tilde{c}\left(s_{1}\right), C_{1}\right)<\eta$.

Interchanging $C_{0}$ and $C_{1}$, if need be, we may assume that $s_{0}<s_{1}$. For any $t \in \mathbb{R}$ we have $\lim _{n \rightarrow \infty} c_{n}\left(t_{n}+t\right)=\tilde{c}(t)$. In particular, $\tilde{c}\left(s_{0}\right)=$ $\lim _{n \rightarrow \infty} c_{n}\left(t_{n}+s_{0}\right)$ and $\tilde{c}\left(s_{1}\right)=\lim _{n \rightarrow \infty} c_{n}\left(t_{n}+s_{1}\right)$. Therefore, for sufficiently large $n$ the inequalities $d\left(c_{n}\left(t_{n}+s_{0}\right), C_{0}\right)<\eta$ and $d\left(c_{n}\left(t_{n}+\right.\right.$ $\left.\left.s_{1}\right), C_{1}\right)<\eta$ hold. Besides, for sufficiently large $n$ we have $0<t_{n}+s_{0}<$ $t_{n}+s_{1}<L_{n}$.

Let $n \in \mathbb{N}$ be any index such that the above conditions hold. Set $L=t_{n}+s_{1}$, and let $c:[0, L] \rightarrow S$ be the restriction of $c_{n}$ to $\left[0, t_{n}+s_{1}\right]$. Then $d(c(0), \partial S) \geq \varepsilon$ and $d\left(c(L), C_{1}\right)<\eta$. But we also have $d\left(c\left(t_{n}+\right.\right.$ $\left.\left.s_{0}\right), C_{0}\right)<\eta$, and $t_{n}+s_{0} \in(0, L)$. By Lemma 5.7, this is impossible.

We will also need the following complement to Lemma 5.1,

Lemma 5.8. For any $\varepsilon>0$ there exists $T=T(\varepsilon)$ such that the following holds. If $c:[0, L] \rightarrow \bar{S}$ is a minimal geodesic such that $d\left(c(0), C_{1}\right) \geq \varepsilon$ and $c(L) \in C_{0}$ then $d\left(c(t), C_{0}\right) \leq \varepsilon$ for all $t \in[T, L]$.

Proof. By Theorem 14 in [Mo] (see also Theorem 6.8 in [Ba1]), there exist minimal geodesics $c_{ \pm}: \mathbb{R} \rightarrow S$ such that $\lim _{t \rightarrow-\infty} d\left(c_{+}(t), C_{0,-}\right)=0$ and $\lim _{t \rightarrow \infty} d\left(c_{+}(t), C_{1,+}\right)=0$, while $\lim _{t \rightarrow-\infty} d\left(c_{-}(t), C_{1,-}\right)=0$ and 
$\lim _{t \rightarrow \infty} d\left(c_{-}(t), C_{0,+}\right)=0$. Hence the geodesics $c_{-}$and $c_{+}$intersect; being minimal, they intersect exactly once. Let $t_{-}, t_{+} \in \mathbb{R}$ be determined by $c_{-}\left(t_{-}\right)=c_{+}\left(t_{+}\right)$. We denote by $S\left(c_{-}, c_{+}\right) \subset S$ the connected component of $S \backslash\left(c_{-}(\mathbb{R}) \cup c_{+}(\mathbb{R})\right)$ whose boundary is $\partial S\left(c_{-}, c_{+}\right)=$ $C_{0} \cup c_{+}\left(\left(-\infty, t_{+}\right]\right) \cup c_{-}\left(\left[t_{-}, \infty\right)\right)$. See Figure 5 .

If $k \in \operatorname{Stab}(S)$ then $c_{+}-k$ and $c_{-}+k$ have the same properties as $c_{+}$and $c_{-}$. If $k$ is not the identity map, then

$$
\cup_{r \in \mathbb{Z}} S\left(c_{-}+r k, c_{+}-r k\right)=S .
$$

A straightforward compactness argument shows that for every $\varepsilon>0$ there exists $r \in \mathbb{Z}$ such that the following holds: If $p \in S$ and $d\left(p, C_{1}\right) \geq$ $\varepsilon$ then there exists $j \in \operatorname{Stab}(S)$ such that

$$
p+j \in S\left(c_{-}+r k, c_{+}-r k\right) .
$$

We set $c_{-}=c_{-}+r k, c_{+}=c_{+}-r k$ and use the preceding statement for $p=c(0)$. Thus, translating $c$ by some $j \in \operatorname{Stab}(S)$, if necessary, we may assume that $c(0) \in S\left(c_{-}, c_{+}\right)$. By the minimality of geodesics $c, c_{-}, c_{+}$ and the inclusions $c(0) \in S\left(c_{-}, c_{+}\right), c(L) \in C_{0}$, we conclude that $c(t) \in$ $S\left(c_{-}, c_{+}\right)$for all $t \in[0, L]$. Now the conditions $\lim _{t \rightarrow-\infty} d\left(c_{+}(t), C_{0,-}\right)=$ 0 and $\lim _{t \rightarrow \infty} d\left(c_{-}(t), C_{0,+}\right)=0$ imply the claim.

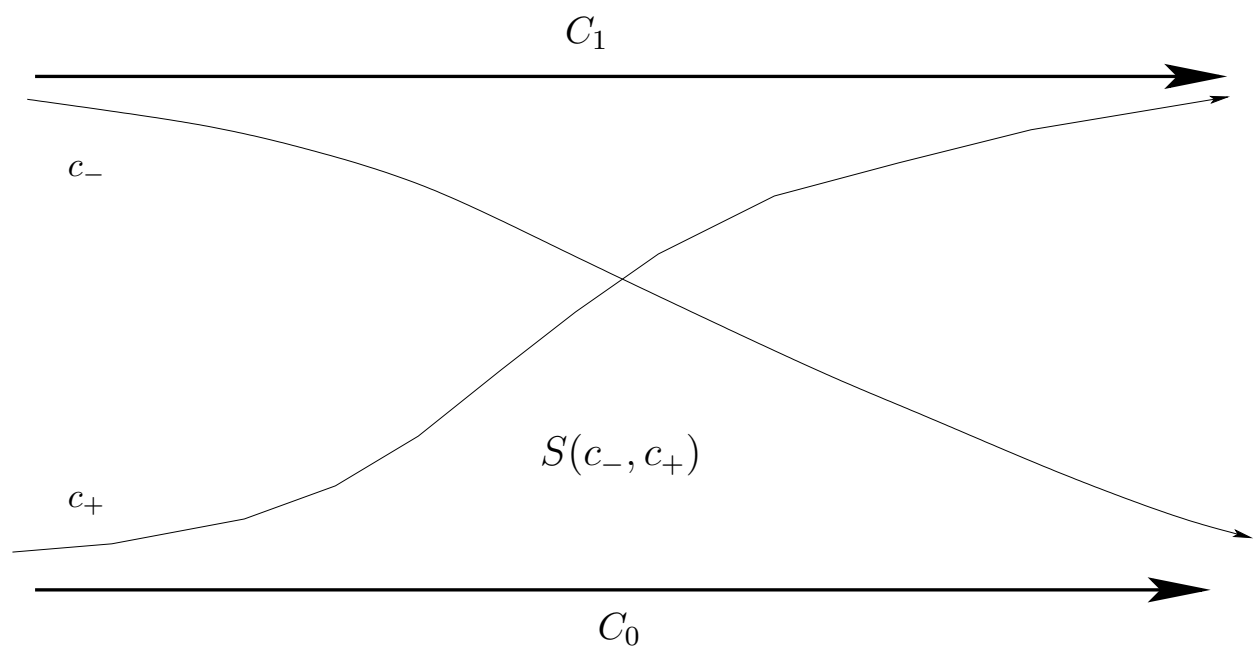

FiguRE 5. Illustrates the proof of Lemma 5.8.

Remark 5.9. The claim of Lemma 5.8 is geometrically obvious, although a formal proof is somewhat cumbersome. For the benefit of the reader, we indicate how to formalize the last step in the preceding 
argument. Using the normal exponential map of $C_{0}$, we represent the ends $c_{+}\left(\left(-\infty,-T_{0}\right]\right)$ and $c_{-}\left(\left[T_{0}, \infty\right)\right)$ of $c_{+}$and $c_{-}$as graphs over $C_{0}$ for sufficiently large $T_{0}$. Then, for sufficiently large $t \in[0, L]$ the point $c(t)$ is located between $C_{0}$ and one of these graphs.

\section{Nonflat two-Dimensional tori are inseCURE}

In this section we prove that every nonflat two-torus has an open set of insecure point pairs. We begin with an auxiliary proposition; it provides a general sufficient condition for a pair of points in a riemannian manifold to be insecure.

Proposition 6.1. Let $p, q$ be points in a complete riemannian manifold $M$. Let $A \subset M$ be a nonempty closed set. Suppose that there exists a sequence of geodesics $c_{n}:\left[0, L_{n}\right] \rightarrow M$ from $p=c_{n}(0)$ to $q=c_{n}\left(L_{n}\right)$ with the following properties:

a) We have $\lim _{n \rightarrow \infty} L_{n}=\infty$;

b) If $n \in \mathbb{N}$ and $t \in\left(0, L_{n}\right)$ then $c_{n}(t) \notin A$;

c) The geodesic $c_{n}$ has no conjugate points in the interval $\left[0, L_{n}\right)$;

d) For every $\varepsilon>0$ there exists $T=T(\varepsilon)>0$ such that for all $n \in \mathbb{N}$ and $T<t<L_{n}-T$ we have $d\left(c_{n}(t), A\right)<\varepsilon$.

Then $p$ and $q$ cannot be blocked away from each other by a finite blocking set.

Proof. In the course of proof we will pass repeatedly from sequences of geodesics to infinite subsequences. In order to avoid cumbersome notation, we will use the following convention. Let $a_{k}$ be an infinite sequence. We will denote its infinite subsequences by $a_{k}$ again, as opposed to, say, $a_{n_{k}}$.

Assume that the claim fails. Then there is a point $z \in M \backslash\{p, q\}$ and infinitely many $n \in \mathbb{N}$ such that $z=c_{n}\left(t_{n}\right)$ for some $t_{n} \in\left(0, L_{n}\right)$. Passing to a subsequence, we assume that for all $n \in \mathbb{N}$ there exist $t_{n} \in\left(0, L_{n}\right)$ such that $z=c_{n}\left(t_{n}\right)$. Set

$$
\varepsilon=\varepsilon(p, q, z, A)=\frac{1}{2} \min (\{d(p, A), d(q, A), d(z, A)\} \backslash\{0\}) .
$$

Let $T=T(\varepsilon)$ be as in condition $\mathrm{d})$. Passing to a subsequence, if need be, and using condition $\mathrm{d})$, we obtain that either $t_{n} \in(0, T)$ or $t_{n} \in\left(L_{n}-T, L_{n}\right)$ for all $n \in \mathbb{N}$. 
Consider first the case $t_{n} \in(0, T)$. Again passing to a subsequence, we assume that $\lim _{n \rightarrow \infty} t_{n}=\bar{t}$ exists, and the geodesics $c_{n}$ converge to a geodesic $c:[0, \infty) \rightarrow M$. Thus, we have

$$
c_{n}\left(t_{n}\right)=z=c(\bar{t})
$$

for all $n \in \mathbb{N}$. Since $c(0)=p$ and $z \neq p$, we conclude that $\bar{t}>0$. By conditions a) and $c$ ), the geodesic $c$ has no conjugate points. This contradicts equation (6), unless we have $c_{n}=\left.c\right|_{\left[0, L_{n}\right]}$ for almost all $n \in \mathbb{N}$.

Suppose this is the case. Then, by condition a), there are positive integers $n<m$ such that $T<L_{n}<L_{m}-T$ and $q=c_{n}\left(L_{n}\right)=$ $c\left(L_{n}\right)=c_{m}\left(L_{n}\right)$. Then, by condition b), $q \notin A$. On the other hand, by condition $\mathrm{d})$, we have $d\left(c_{m}\left(L_{n}\right), A\right)<\varepsilon$. This contradicts our choice of $\varepsilon \leq d(q, A)$. Thus, we have arrived at a contradiction.

It remains to consider the case $t_{n} \in\left(L_{n}-T, L_{n}\right)$. Observe that our setting is symmetric with respect to the interchange of $p$ and $q$ and simultaneous reversal of the time direction. This symmetry flips the two cases at hand. Thus, the assumption $t_{n} \in\left(L_{n}-T, L_{n}\right)$ leads to a contradiction as well.

Our next result yields the existence of a nonempty open set of insecure point pairs in a nonflat two-torus.

Theorem 6.2. Let $T^{2}$ be a nonflat riemannian two-torus. Let $Z \subset T^{2}$ be an admissible cylinder, as introduced in section 4 . Then every pair $(p, q) \in Z \times \bar{Z}$ is insecure.

Proof. We apply Proposition 6.1 to our torus, with $A=\partial Z$. Let $S$ be an admissible strip corresponding to $Z$; let $P \in S, Q \in \bar{S}$ be such that $\pi(P)=p, \pi(Q)=q$. Let $k \in \operatorname{Stab}(S)$ be as in section 4 . For every $n \in \mathbb{N}$ we choose a minimal geodesic $\tilde{c}_{n}:\left[0, L_{n}\right] \rightarrow \bar{S}$ from $\tilde{c}_{n}(0)=P$ to $\tilde{c}_{n}\left(L_{n}\right)=Q+n k$. Set $c_{n}=\pi \circ \tilde{c}_{n}$. By definition, this is a sequence of geodesics $c_{n}:\left[0, L_{n}\right] \rightarrow T^{2}$ from $p$ to $q$.

Next, we check that the assumptions of Proposition 6.1 are satisfied. Assumptions a) and c) are satisfied by construction. Assumption b) is satisfied because $p \in Z$ and the set $A=\partial Z$ is totally geodesic. Assumption d) follows from Lemma 5.1 when $q \in Z$ and from Lemma 5.8 when $q \in \partial Z$.

Remark 6.3. The preprint [WKH] gives an argument to show the insecurity for pairs $(p \in Z, q \in \partial Z)$. This observation is not contained in [BaG08] which is a preliminary version of the present paper. 
Theorem 6.4. A two-dimensional riemannian torus is secure if and only if it is flat.

Proof. By [Gut05] or [GS06], a flat torus is secure. Theorem 6.2 implies the existence of insecure point pairs in any nonflat two-torus.

We point out that the flat tori are distinguished amongst all riemannian two-tori by the security of pairs $y=x$.

Corollary 6.5. A two-dimensional riemannian torus is flat if and only if all pairs $(x, x)$ are secure.

Proof. It suffices to show that a nonflat riemannian two-torus contains at least one point that cannot be blocked away from itself. Let $Z$ be an admissible cylinder. Then any point $x \in Z$ cannot be blocked away from itself.

\section{Blocking AND INSECURITY FOR TORI OF REVOLUTION}

By a two-torus of revolution we will mean the cartesian square $T^{2}$ of the standard circle $T=\mathbb{R} / \mathbb{Z}$ with the riemannian metric

$$
d s^{2}=f^{2}(y) d x^{2}+d y^{2} .
$$

The somewhat more general $T$-invariant riemannian tori

$$
d s^{2}=f^{2}(y) d x^{2}+g^{2}(y) d y^{2},
$$

reduce to the torus of revolution equation (77) by a change of variables.

Thus, our torus of revolution is determined by the positive function $f: T \rightarrow \mathbb{R}_{+}$. To emphasize the dependence on $f$ we will sometimes denote the riemannian torus in equation (7) by $T_{f}^{2}$. We will assume that $f$ is strictly positive and infinitely differentiable.

Our analysis is based on the symmetries of $T_{f}^{2}$. The riemannian metric in equation (7) is invariant with respect to the action of $T$ on $T^{2}$ by $(x, y) \mapsto(x+a, y)$. Besides rotations $\rho_{a}(x)=x+a \bmod 1$, the isometry group of $T$ contains reflections. With each $a \in T$ we associate the reflection $\sigma_{a}(x)=2 a-x \bmod 1$. Then $\sigma_{a}$ is orientation reversing; it has two fixed points: $a, a+\frac{1}{2}$.

Proposition 7.1. Let $f: T \rightarrow \mathbb{R}_{+}$be a smooth, positive function. Suppose that i) it has a unique minimum, say at the point $a \in T$; ii) it is invariant under the reflection $\sigma_{a}$.

Then all pairs $\{(p, a),(q, a)\} \in T_{f}^{2} \times T_{f}^{2}$ are secure. All other pairs of points in $T_{f}^{2}$ are insecure. 
Proof. Let $F, G$ be transformations of $T$. We denote by $F \times G$ the transformation of $T^{2}$ given by $(x, y) \mapsto(F(x), G(y))$.

For arbitrary points $p, q \in T$ let $F_{p, q}: T \rightarrow T$ be the unique reflection interchanging $p$ and $q$. If $r \in T$ is any of the two midpoints between $p$ and $q$, then $F_{p, q}=\sigma_{r}$. We set

$$
\varphi_{p, q}=F_{p, q} \times \sigma_{a} .
$$

Then $\varphi_{p, q}: T^{2} \rightarrow T^{2}$ is an involution: $\varphi_{p, q}^{2}=\mathrm{Id}$; it has exactly 4 fixed points.

The coordinate vector fields $\partial / \partial x, \partial / \partial y$ on $T^{2}$ yield global coordinates on the tangent bundle to $T_{f}^{2}$. Let $X$ be a tangent vector to $T^{2}$. The unique decomposition

$$
X=\xi \frac{\partial}{\partial x}+\eta \frac{\partial}{\partial y}
$$

defines the isomorphism $X \mapsto((x, y),(\xi, \eta))$ of the tangent bundle and $T^{2} \times \mathbb{R}^{2}$. If the base point of a tangent vector $X$ is clear from the context, we will identify $X$ with $(\xi, \eta) \in \mathbb{R}^{2}$. Let $H: T^{2} \rightarrow T^{2}$ be a diffeomorphism. Our isomorphism identifies the differentials $D_{(x, y)} H$ with $2 \times 2$ matrices. By equation (9), we have

$$
D_{(x, y)} \varphi_{p, q}=-\mathrm{Id}
$$

identically on $T^{2}$.

From now on, we view $T^{2}$ as the riemannian torus of revolution $T_{f}^{2}$. Let $y \in T$. By analogy with the round euclidean torus, we define the circles of latitude as $L_{y}=T \times\{y\} \subset T_{f}^{2}$.

Let $X$ be the tangent vector with the base point $(x, y)$ and the representation $X=(\xi, \eta)$. The function on the tangent bundle given by

$$
F(X)=f^{2}(y) \xi
$$

is the Clairaut integral for the geodesic flow on the tangent bundle of $T_{f}^{2}\left[\mathrm{Kl}\right.$. Let $c: \mathbb{R} \rightarrow T_{f}^{2}$ be a geodesic. Set $c(t)=(x(t), y(t)), \dot{c}(t)=$ $(\dot{x}(t), \dot{y}(t))$. Let $t_{1} \neq t_{2}$ be such that $y\left(t_{1}\right)=y\left(t_{2}\right)$. Then, from equations (7), (11)

$$
\dot{x}\left(t_{1}\right)=\dot{x}\left(t_{2}\right),\left|\dot{y}\left(t_{1}\right)\right|=\left|\dot{y}\left(t_{2}\right)\right| \text {. }
$$

Suppose now that $c(0) \in L_{a}$. Then, by equations (7) and (11)

$$
\min _{t \in \mathbb{R}}|\dot{y}(t)|=|\dot{y}(0)| \text {. }
$$

By equation (13), the geodesics $c(t)=(x(t), y(t))$ in $T_{f}^{2}$ that intersect the circle $L_{a}$ satisfy the following dichotomy: Either $\dot{y}(t) \equiv 0$ or $\dot{y}(t)$ does not change sign. In the former case, the trace of $c$ is $L_{a}$. In 
the latter case, the geodesic $c$ winds around $T_{f}^{2}$ crossing every circle of latitude transversally, as opposed to oscillating between two circles of latitude. Thus, for the geodesics $c: \mathbb{R} \rightarrow T_{f}^{2}$ intersecting the circle $L_{a}$, equation (12) becomes

$$
\dot{x}\left(t_{1}\right)=\dot{x}\left(t_{2}\right), \dot{y}\left(t_{1}\right)=\dot{y}\left(t_{2}\right) .
$$

Let $\lambda$ be the free homotopy class of the circles of latitude. By condition i) on $f$, the closed curve $L_{a} \subset T_{f}^{2}$ is the unique minimal geodesic in $\lambda$. In view of Theorem 6.2, it suffices to show that any pair of points in $L_{a}$ is secure. To simplify notation, we will use the shorthand $\{(p, a),(q, a)\}=\{p, q\} \in L_{a} \times L_{a}$. For any torus of revolution $T_{f}^{2}$ the transformations $F_{p, q} \times \mathrm{Id}: T_{f}^{2} \rightarrow T_{f}^{2}$ are isometries. By assumption ii), the transformation Id $\times \sigma_{a}: T_{f}^{2} \rightarrow T_{f}^{2}$ is also an isometry of $T_{f}^{2}$. Thus, the involutions $\varphi_{p, q}: T_{f}^{2} \rightarrow T_{f}^{2}$ from equation (9) are isometries.

We denote by $G_{p, q}$ the set of geodesics $c: \mathbb{R} \rightarrow T_{f}^{2}$ such that for some $t_{p}<t_{q}$ we have $c\left(t_{p}\right)=p, c\left(t_{q}\right)=q$. Restricting these geodesics to $\left[t_{p}, t_{q}\right]$ and taking their traces, we obtain the set $\Gamma_{p, q}$ of geodesic segments connecting $p$ and $q$. Note that $\Gamma_{q, p}=\Gamma_{p, q}$. By equation (9), $\varphi_{p, q}: G_{p, q} \rightarrow G_{q, p}$, and hence $\varphi_{p, q}\left(\Gamma_{p, q}\right)=\Gamma_{p, q}$. We will now show that actually $\varphi_{p, q}$ maps every geodesic segment $c \in G_{p, q}$ to itself.

Let $c \in G_{p, q}$. By equation (14), $\dot{c}\left(t_{p}\right)=\dot{c}\left(t_{q}\right)$. In view of equation (10), every geodesic geodesic segment connecting $p$ and $q$ is invariant under $\varphi_{p, q}$. Since $\varphi_{p, q}$ interchanges $p$ and $q$, it fixes the midpoint of each $\gamma \in \Gamma_{p, q}$.

Thus, the midpoint, say $m(\gamma)$, of any $\gamma \in \Gamma_{p, q}$ is one of the four fixed points of $\varphi_{p, q}$. It may happen that $m(\gamma)=p$. Then $\gamma$ is a multiple of a prime geodesic segment $\beta \in \Gamma_{p, q}$. Applying the same argument to $\beta$, we conclude that the set of fixed points of $\varphi_{p, q}$ minus the set $\{p, q\}$ is a blocking set for $\Gamma_{p, q}$.

Remark 7.2. A pair $\{p, q\}$ in a riemannian manifold is midpoint secure if the set of midpoints of geodesics $\gamma \in \Gamma_{p, q}$ is finite. A manifold is midpoint secure if every pair in $M$ is midpoint secure. Flat manifolds are midpoint secure. Secure pairs in compact symmetric spaces are midpoint secure [GS06]. By our proof of Proposition [7.1, a pair in a torus of revolution is either insecure or midpoint secure. These examples seem to suggest that security is equivalent to the midpoint security. This is false, however. P. Herreros [Her gave an example of a riemannian two-sphere with a large family of point pairs that are secure but not midpoint secure. 
Corollary 7.3. Let $T^{2}=T^{2}(r, R) \subset \mathbb{R}^{3}$ be a round euclidean torus of revolution. See Example 3.1. Let $E_{\text {in }} \subset T^{2}$ be the inner equator. Then a point pair $\{p, q\} \in T^{2} \times T^{2}$ is secure if and only if $\{p, q\} \in E_{\text {in }} \times E_{\text {in }}$. The pairs in $E_{\text {in }}$ are, in fact, midpoint secure.

Proof. We will refer to $T^{2}(r, R) \subset \mathbb{R}^{3}$ as the round euclidean torus. In the standard angular coordinates $0 \leq \alpha, \beta \leq 2 \pi$ the round euclidean torus has the metric

$$
d s^{2}=(R-r \cos \beta)^{2} d \alpha^{2}+r^{2} d \beta^{2} .
$$

Since the round euclidean torus is a special case of the torus in equation (8), we reduce it to a torus of revolution $T_{f}^{2}$ of equation (7). In this representation we have $f(y)=R-r \cos (2 \pi y)$, up to a constant factor. This function has a unique minimum, $f(0)=R-r$, and is invariant under the reflection $\sigma(y)=-y \bmod 1$. Hence, Proposition 7.1 and Remark 7.2 apply.

\section{INSECURITY FOR ALL POINT PAIRS IN A TWO-DIMENSIONAL TORUS}

In the preceding section we saw that there are nonflat two-tori with some secure point pairs. Now we will prove that such tori are exceptional. We will exhibit a $C^{2}$-open and $C^{\infty}$-dense subset $\mathcal{G}_{\text {tot }}$ of the space of riemannian metrics on the two-torus such that for metrics in $\mathcal{G}_{\text {tot }}$ every pair $(p, q) \in T^{2} \times T^{2}$ is insecure.

We will recall a few well known facts about homotopically minimal geodesics on an arbitrary riemannian two-torus $\left(T^{2}, g\right)$. First, we explain what we mean by the homological direction of a homotopically minimal geodesic $c: \mathbb{R} \rightarrow T^{2}$. If $\tilde{c}: \mathbb{R} \rightarrow \mathbb{R}^{2}$ is a lift of $c$, then there is a strip bounded by two parallel lines in $\mathbb{R}^{2}$ that contains $\tilde{c}$. The minimality of $c$ implies that $\tilde{c}$ is proper and separates the boundary components of the strip. This was first proven by G.A. Hedlund [He]. See also [Ba1, Theorem 6.5. Moreover, $\tilde{c}$ determines an orientation for the parallel lines in the strip. This oriented direction can be viewed as an element in the quotient of $\mathbb{R}^{2} \backslash\{0\}$ by the multiplicative action of $\mathbb{R}_{+}$. Representing $T^{2}$ as $\mathbb{R}^{2} / \mathbb{Z}^{2}$, as usual, we identify $\mathbb{Z}^{2} \subset \mathbb{R}^{2}$ with $H_{1}\left(T^{2}, \mathbb{Z}\right)$ and $\mathbb{R}^{2}$ with $H_{1}\left(T^{2}, \mathbb{R}\right)$.

The homological direction $[h] \in\left(H_{1}\left(T^{2}, \mathbb{R}\right) \backslash\{0\}\right) / \mathbb{R}_{+}$of the homotopically minimal geodesic $c$ is the oriented direction of a strip that contains a lift of $c$. Let $c$ be a periodic geodesic of minimal length in its free homotopy class. Then $c$ is homotopically minimal, by Theorem 2.3 . Let $h=h(c)$ be its homology class. Then the homological direction of $c$ is the image of $h(c) \in H_{1}\left(T^{2}, \mathbb{Z}\right)$ in the quotient $\left(H_{1}\left(T^{2}, \mathbb{R}\right) \backslash\{0\}\right) / \mathbb{R}_{+}$. 
Alternatively, one can define the homological direction of a homotopically minimal geodesic as follows. Let $c: \mathbb{R} \rightarrow T^{2}$ be one. Let $s_{i}, t_{i} \in \mathbb{R}, i \in \mathbb{N}$, be two infinite sequences such that $s_{i}<t_{i}$ and $t_{i}-s_{i} \rightarrow \infty$. Let $\gamma_{i}, i \in \mathbb{N}$, be arbitrary curves from $c\left(t_{i}\right)$ to $c\left(s_{i}\right)$ such that their lengths are uniformly bounded. We will denote by $\alpha * \beta$ the concatenation of curves $\alpha$ and $\beta$ whenever it is defined. Let $h_{i} \in H_{1}\left(T^{2}, \mathbb{R}\right)$ be the homology class of the closed curve $\left.c\right|_{\left[s_{i}, t_{i}\right]} * \gamma_{i}$. Then, for all but a finite number of indices, we have $h_{i} \neq 0$, defining the direction $\left[h_{i}\right] \in\left(H_{1}\left(T^{2}, \mathbb{R}\right) \backslash\{0\}\right) / \mathbb{R}_{+}$. Moreover, as $i \rightarrow \infty$, the sequence $\left[h_{i}\right]$ converges; the limit $[h]=\lim _{i \rightarrow \infty}\left[h_{i}\right]$ is the homological direction of $c$. See Sch for a similar concept.

Let $S$ be an oriented surface. Let $v_{1}, v_{2}$ be linearly independent tangent vectors with the same base point in $S$. We set $\varepsilon\left(v_{1}, v_{2}\right)=1$ if the pair $v_{1}, v_{2}$ is positively oriented, and $\varepsilon\left(v_{1}, v_{2}\right)=-1$ otherwise. Let $\left[h_{1}\right],\left[h_{2}\right] \in\left(H_{1}\left(T^{2}, \mathbb{R}\right) \backslash\{0\}\right) / \mathbb{R}_{+}$. Let $h_{i} \in\left[h_{i}\right]$ for $i=1,2$. Then the sign of the homological intersection $h_{1} \cdot h_{2}$ does not depend on the choices of $h_{i} \in\left[h_{i}\right]$. Hence, we will use the notation $\operatorname{sgn}\left(h_{1} \cdot h_{2}\right) \in$ $\{0, \pm 1\}$.

We will need the following property of homotopically minimal geodesics on a two-torus.

Lemma 8.1. Let $c_{1}: \mathbb{R} \rightarrow T^{2}, c_{2}: \mathbb{R} \rightarrow T^{2}$ be homotopically minimal geodesics. Let $\left[h_{1}\right],\left[h_{2}\right] \in\left(H_{1}\left(T^{2}, \mathbb{R}\right) \backslash\{0\}\right) / \mathbb{R}_{+}$be their homological directions.

Suppose that $\operatorname{sgn}\left(h_{1} \cdot h_{2}\right) \neq 0$. Then for any $t_{1}, t_{2} \in \mathbb{R}$ such that $c_{1}\left(t_{1}\right)=c_{2}\left(t_{2}\right)$, we have

$$
\varepsilon\left(\dot{c}_{1}\left(t_{1}\right), \dot{c}_{2}\left(t_{2}\right)\right)=\operatorname{sgn}\left(h_{1} \cdot h_{2}\right) .
$$

Proof. Let $\tilde{c}_{i}: \mathbb{R} \rightarrow \mathbb{R}^{2}, i=1,2$, be the lifts of $c_{i}$ such that $\tilde{c}_{1}\left(t_{1}\right)=$ $\tilde{c}_{2}\left(t_{2}\right)$. We will prove that $\varepsilon\left(\dot{\tilde{c}}_{1}\left(t_{1}\right), \dot{\tilde{c}}_{2}\left(t_{2}\right)\right)=\operatorname{sgn}\left(h_{1} \cdot h_{2}\right)$.

We identify $H_{1}\left(T^{2}, \mathbb{R}\right)$ with $\mathbb{R}^{2}$, as always, and set $l_{i}=\left\{t h_{i}: t \in \mathbb{R}\right\}$ where $i=1,2$. Note that $l_{1}, l_{2}$ are oriented straight lines in $\mathbb{R}^{2}$. For $(s, t) \in \mathbb{R} \times[0,1]$ and $i=1,2$, set $H_{i}(s, t)=(1-t) \tilde{c}_{i}(s)+t s h_{i}$. Then $H_{i}: \mathbb{R} \times[0,1] \rightarrow \mathbb{R}^{2}$ is a homotopy between $\tilde{c}_{i}$ and the line $l_{i}$. Set $K=\left\{(s, t) \in \mathbb{R} \times[0,1]: H_{1}(s, t) \in l_{2}\right\}$. By remarks in the beginning of this section, $K$ is a compact subset of $\mathbb{R} \times[0,1]$. Standard arguments from differential topology show that the oriented intersection numbers $\#\left(\tilde{c}_{1}, \tilde{c}_{2}\right), \#\left(\tilde{c}_{1}, l_{2}\right), \#\left(l_{1}, \tilde{c}_{2}\right)$ are defined and satisfy

$$
\#\left(\tilde{c}_{1}, \tilde{c}_{2}\right)=\#\left(\tilde{c}_{1}, l_{2}\right)=\#\left(l_{1}, l_{2}\right) .
$$

\footnotetext{
${ }^{3}$ See, e. g., Hi], Chapter 5.
} 
We obviously have

$$
\#\left(l_{1}, l_{2}\right)=\operatorname{sgn}\left(h_{1} \cdot h_{2}\right)
$$

Since $\tilde{c}_{1}, \tilde{c}_{2}$ are minimal geodesics, they intersect exactly once, and transversely, at the point $\tilde{c}_{1}\left(t_{1}\right)=\tilde{c}_{2}\left(t_{2}\right)$. Hence

$$
\#\left(\tilde{c}_{1}, \tilde{c}_{2}\right)=\varepsilon\left(\dot{\tilde{c}}_{1}\left(t_{1}\right), \dot{\tilde{c}}_{2}\left(t_{2}\right)\right) .
$$

Equations (16) - (18) imply our claim.

Corollary 8.2. Let $h_{1}, h_{2}$ be prime elements in the lattice of integer classes in $H_{1}\left(T^{2}, \mathbb{R}\right)$. Let $c_{1}, c_{2}: \mathbb{R} \rightarrow T^{2}$ be minimal periodic geodesics in the classes $h_{1}, h_{2}$ respectively. Suppose that $h_{1} \cdot h_{2} \neq 0$. Then

$$
\operatorname{card}\left(c_{1}(\mathbb{R}) \cap c_{2}(\mathbb{R})\right)=\left|h_{1} \cdot h_{2}\right| .
$$

Proof. Denote by $L_{i}$ the minimal period of $c_{i}$. Since $c_{1}$ and $c_{2}$ intersect transversely, we have

$$
h_{1} \cdot h_{2}=\sum_{\left(t_{1}, t_{2}\right)} \varepsilon\left(\dot{c}_{1}\left(t_{1}\right), \dot{c}_{2}\left(t_{2}\right)\right)
$$

where the sum is over all pairs $\left(t_{1}, t_{2}\right) \in\left[0, L_{1}\right) \times\left[0, L_{2}\right)$ such that $c_{1}\left(t_{1}\right)=c_{2}\left(t_{2}\right)$. By Lemma 8.1

$$
\left|h_{1} \cdot h_{2}\right|=\operatorname{card}\left\{\left(t_{1}, t_{2}\right) \in\left[0, L_{1}\right) \times\left[0, L_{2}\right): c_{1}\left(t_{1}\right)=c_{2}\left(t_{2}\right)\right\} .
$$

Since $h_{1}, h_{2} \in H_{1}\left(T^{2}, \mathbb{R}\right)$ are prime, the curves $c_{1}:\left[0, L_{1}\right) \rightarrow T^{2}$ and $c_{2}:\left[0, L_{2}\right) \rightarrow T^{2}$ are injective. Hence, for every $p \in c_{1}(\mathbb{R}) \cap c_{2}(\mathbb{R})$ there is exactly one pair $\left(t_{1}, t_{2}\right) \in\left[0, L_{1}\right) \times\left[0, L_{2}\right)$ such that $p=c_{1}\left(t_{1}\right)=$ $c_{2}\left(t_{2}\right)$. Now our claim follows directly from equation (19).

We will investigate riemannian metrics on the two-torus. We denote by $\mathcal{G}_{1}, \mathcal{G}_{2}$ the sets of riemannian metrics on $T^{2}$ satisfying the conditions $G_{1}, G_{2}$ respectively:

$\left(G_{1}\right)$ There exists a homology class $h \in H_{1}\left(T^{2}, \mathbb{R}\right) \backslash\{0\}$ such that $T^{2}$ does not admit a foliation by homotopically minimal geodesics with the homological direction $[h]$;

$\left(G_{2}\right)$ There exist integral homology classes $h_{1}, h_{2} \in H_{1}\left(T^{2}, \mathbb{R}\right)$ such that $h_{1} \cdot h_{2}=1$ and for each $i=1,2$ there is a unique minimal periodic geodesic in the class $h_{i}$.

We will now show that for $g \in \mathcal{G}_{1} \cap \mathcal{G}_{2}$ any point pair in $\left(T^{2}, g\right)$ is insecure. 
Theorem 8.3. Let $\left(T^{2}, g\right)$ be a riemannian torus. If the metric $g$ satisfies conditions $\left(G_{1}\right)$ and $\left(G_{2}\right)$, then every pair of points in $\left(T^{2}, g\right)$ is insecure.

Proof. Suppose the claim is false, and let $(p, q)$ be a secure pair of points in our torus. Let $h_{1}, h_{2} \in H_{1}\left(T^{2}, \mathbb{R}\right)$ be as in condition $\left(G_{2}\right)$; we denote by $c_{1} \in h_{1}, c_{2} \in h_{2}$ the unique minimal periodic geodesics in these classes. Then $Z_{i}=T^{2} \backslash c_{i}(\mathbb{R})$ are admissible cylinders. By Theorem 6.2 , both points $p, q$ belong to the sets $c_{1}(\mathbb{R}), c_{2}(\mathbb{R})$. Thus, $\{p, q\} \subset c_{1}(\mathbb{R}) \cap c_{2}(\mathbb{R})$.

By Corollary [8.2, the geodesics $c_{1}, c_{2}$ intersect at a unique point. Thus $p=q$, and $\{p\}=c_{1}(\mathbb{R}) \cap c_{2}(\mathbb{R})$. We will now study the geodesics in $\left(T^{2}, g\right)$ passing through $p$.

In view of Theorem 6.2, we know that for any free homotopy class of prime periodic geodesics, there exists a minimal geodesic in this class passing through $p$. Taking limits of these geodesics, we conclude that for every $h \in H_{1}\left(T^{2}, \mathbb{R}\right) \backslash\{0\}$ there exists a homotopically minimal geodesic with the homological direction $[h]$ passing through $p$. By Theorem 6.7 and Theorem 6.9 in [Ba1, this geodesic is uniquely determined by $[h]$. We denote it by $c([h])$. Let $U T_{p}^{2} \subset T_{p}^{2}$ be the set of unit tangent vectors at $p$. Let $\Phi([h]) \in U T_{p}^{2}$ be the tangent vector at $p$ to the geodesic $c([h])$. This defines a map

$$
\Phi:\left(H_{1}\left(T^{2}, \mathbb{R}\right) \backslash\{0\}\right) / \mathbb{R}_{+} \rightarrow U T_{p}^{2} .
$$

By Theorem VII in [He], or by Remark (2) on page 32 in [Ba1, there is a uniform bound on the widths of the strips in $\mathbb{R}^{2}$ containing lifts of homotopically minimal geodesics. It follows that the map $\Phi$ is continuous. Since it is also injective, we conclude that $\Phi$ is a homeomorphism. Therefore, every geodesic in $\left(T^{2}, g\right)$ passing through $p$ is homotopically minimal.

We have shown that $p$ is a pole for the riemannian torus $\left(T^{2}, g\right)$. This means that any geodesic passing through $p$ has no points conjugate to $p$. Now we use the following result of $\mathrm{Ba} 4$ : If $\left(T^{2}, g\right)$ has a pole, then for every $[h] \in\left(H_{1}\left(T^{2}, \mathbb{R}\right) \backslash\{0\}\right) / \mathbb{R}_{+}$the torus admits a foliation by minimal geodesics with the homological direction $[h]$. Thus, the riemannian torus $\left(T^{2}, g\right)$ does not satisfies condition $\left(G_{1}\right)$. We have arrived at a contradiction.

We have shown that for riemannian tori $\left(T^{2}, g\right)$, where $g \in \mathcal{G}_{1} \cap$ $\mathcal{G}_{2}$, every pair of points is insecure. Recall that a closed geodesic $\gamma$ 
is nondegenerate if the linearized Poincaré map for $\gamma$ does not have eigenvalue 1.

Let $\mathcal{G}_{3} \subset \mathcal{G}_{2}$ be the set of tori $\left(T^{2}, g\right)$ satisfying the following strengthening of condition $\left(G_{2}\right)$ :

$\left(G_{3}\right)$ There exist integral homology classes $h_{1}, h_{2} \in H_{1}\left(T^{2}, \mathbb{R}\right)$ such that $h_{1} \cdot h_{2}=1$ and there is a unique minimal periodic geodesic in the class $h_{i}, i=1,2$. Moreover, these geodesics are nondegenerate.

Proposition 8.4. The set $\mathcal{G}_{1} \cap \mathcal{G}_{3}$ of riemannian metrics on the twotorus satisfying conditions $\left(G_{1}\right)$ and $\left(G_{3}\right)$ is $C^{2}$-open and $C^{\infty}$-dense in the space of riemannian metrics.

Our proof of Proposition 8.4 is based on the following two lemmas.

Lemma 8.5. The set $\mathcal{G}_{1}$ of metrics on the two-torus satisfying condition $\left(G_{1}\right)$ is $C^{2}$-open and $C^{\infty}$-dense.

Proof. Let $h \in H_{1}\left(T^{2}, \mathbb{R}\right) \backslash\{0\}$ be an integral class. Denote by $\mathcal{G}_{1}(h)$ the set of metrics that do not admit a foliation by homotopically minimal geodesics with homological direction $[h]$. We will prove first that $\mathcal{G}_{1}(h)$ is $C^{\infty}$-dense. Since $\mathcal{G}_{1}(h) \subset \mathcal{G}_{1}$, this will prove that $\mathcal{G}_{1}$ is $C^{\infty}$-dense.

Let $g$ be a metric in the complement $\mathcal{G}_{1}(h)^{c}$ of $\mathcal{G}_{1}(h)$, i. e., there exists a foliation $\mathcal{F}$ of $T^{2}$ by homotopically minimal geodesics (with respect to $g$ ) of homological direction $[h]$. Then $\mathcal{F}$ has the following structure: i) The torus contains a nonempty closed subset foliated by periodic geodesics in $\mathcal{F}$; ii) The complement to this set is either empty or it is a disjoint union of admissible cylinders foliated by geodesics in $\mathcal{F}$ that are heteroclinic to the boundary geodesics of the cylinder. See, e. g., Ba1, Theorem 6.6 and Theorem 6.7.

We will now show that if not all of $T^{2}$ is foliated by periodic geodesics in $\mathcal{F}$, then $g$ can be $C^{\infty}$-approximated by a sequence in $\mathcal{G}_{1}(h)$. Let $Z \subset T^{2}$ be an admissible cylinder foliated by heteroclinic geodesics in $\mathcal{F}$. We choose two minimal heteroclinic geodesics $c_{-}: \mathbb{R} \rightarrow Z$ and $c_{+}: \mathbb{R} \rightarrow Z$, one for each of the two possibilities; see Lemma 5.4. Then $U=Z \backslash\left(c_{-}(\mathbb{R}) \cup c_{+}(\mathbb{R})\right)$ is a nonempty open subset of $Z$. We choose $p \in U$ and a sequence $g_{k}$ of riemannian metrics such that a) $g_{k} \geq g$, b) $\left.g_{k}\right|_{p}>\left.g\right|_{p}$, and c) $\left.g_{k}\right|_{T^{2} \backslash U}=\left.g\right|_{T^{2} \backslash U}$. It follows that each of the tori $\left(T^{2}, g_{k}\right)$ has neither a heteroclinic nor a periodic homotopically minimal geodesic with homological direction $[h]$ passing through $p$. Hence $g_{k} \in$ $\mathcal{G}_{1}(h)$ for all $k \in \mathbb{N}$. This proves that $\mathcal{G}_{1}(h)$ is $C^{\infty}$-dense in the set of all metrics on $T^{2}$ that do not admit a foliation by periodic minimal geodesics of direction $[h]$. 
It remains to prove that every metric that admits such a foliation can be $C^{\infty}$-approximated by metrics that do not admit such a foliation. This can be shown by a perturbation argument, which is similar, but simpler, than the argument in the first part of the proof.

To prove that $\mathcal{G}_{1}$ is open in the $C^{2}$-topology, it suffices to show that $\mathcal{G}_{1}(h)^{c}$ is $C^{2}$-closed for every $h \in H_{1}\left(T^{2}, \mathbb{R}\right) \backslash\{0\}$. This fact is closely related to the convergence properties for invariant circles of monotone twist maps. For the benefit of the reader, we will outline a proof.

Let $g_{i} \in \mathcal{G}_{1}(h)^{c}$ be an infinite sequence such that $g_{i} \rightarrow g$ in the $C^{2}$ topology. Let $\mathcal{F}_{i}, i=1,2, \ldots$, be the corresponding foliations of $T^{2}$. Denote by $U_{i}$ the vector field formed by the unit tangent vectors on $\left(T^{2}, g_{i}\right)$ tangent to the foliation $\mathcal{F}_{i}$. Then the sequence $U_{i}$ is equicontinuous, since the vector fields $U_{i}$ are uniformly lipschitz. See [So] or [Mat. By the Arzela-Ascoli theorem, there is a subsequence of vector fields $U_{i}$ that converges to a $g$-unit tangent vector field $U$. The integral curves of $U$ form a foliation $\mathcal{F}$ of $\left(T^{2}, g\right)$ by homotopically $g$-minimal geodesics. The widths of the parallel strips containing the lifts of the geodesics in the foliations $\mathcal{F}_{i}$ to $\mathbb{R}^{2}$ can be chosen uniformly bounded. See, e. g., the proof of Remark (2) on p. 32 in [Ba1, 4 It follows that the geodesics in $\mathcal{F}$ have homological direction $[h]$. Hence, $g \in \mathcal{G}_{1}(h)^{c}$.

Lemma 8.6. The set $\mathcal{G}_{3}$ of riemannian metrics on the two-torus satisfying condition $\left(G_{3}\right)$ is $C^{2}$-open and $C^{\infty}$-dense.

Proof. First, we will show that $\mathcal{G}_{3}$ is $C^{2}$-open. Suppose that $g$ satisfies condition $\left(G_{3}\right)$ for integer classes $h_{1}, h_{2} \in H_{1}\left(T^{2}, \mathbb{R}\right)$ with $h_{1} \cdot h_{2}=1$. Let $c_{1}, c_{2}$ be the unique $g$-minimal periodic geodesics in the classes $h_{1}, h_{2}$ respectively. Let $g_{k}, k \geq 1$, be a sequence of riemannian metrics on $T^{2}$ converging to $g$ in the $C^{2}$-topology. We will show that all but a finite number of the metrics $g_{k}$ satisfy condition $\left(G_{3}\right)$ with the classes $h_{1}, h_{2}$.

Suppose first that an infinite subset of these metrics has several minimal periodic geodesics in one of the homology classes, say $h_{1}$. Relabelling the indices, we obtain a sequence of riemannian metrics $g_{k}$ converging to $g$ and such that there exist distinct $g_{k}$-minimal periodic geodesics $c_{k}^{\prime}$ and $c_{k}^{\prime \prime}$ in the class $h_{1}$. They are distinct in the sense that $c_{k}^{\prime}(\mathbb{R}) \neq c_{k}^{\prime \prime}(\mathbb{R})$. With an appropriate reparameterization, both sequences of geodesics converge to the $g$-minimal geodesic $c_{1}$. By the

\footnotetext{
${ }^{4}$ Alternatively, we can use the order of the $\mathbb{Z}^{2}$-translates of such lifts to determine the direction. See, e. g., $\mathrm{We}$ or $\mathrm{Ba3}$.
} 
implicit function theorem, the linearized Poincaré map for $c$ has an eigenvalue 1, contrary to our assumption.

Deleting a finite number of indices, we assume without loss of generality that for all $k \in \mathbb{N}$ the metrics $g_{k}$ have unique minimal periodic geodesics $c_{k}^{(1)}, c_{k}^{(2)}$ in the classes $h_{1}, h_{2}$ respectively. We claim that for all but a finite number of indices the geodesics $c_{k}^{(1)}, c_{k}^{(2)}$ are nondegenerate. We may assume that we have the convergence $c_{k}^{(1)} \rightarrow c_{1}, c_{k}^{(2)} \rightarrow c_{2}$. Since the geodesic flows of $\left(T^{2}, g_{i}\right)$ converge in the $C^{1}$-topology to the geodesic flow of $\left(T^{2}, g\right)$, and the limit geodesics are nondegenerate, we obtain the claim.

We have shown that $\mathcal{G}_{3}$ is $C^{2}$-open. We will now prove that $\mathcal{G}_{3}$ is $C^{\infty}$-dense. Let $g$ be any $C^{\infty}$ metric on the torus. Let $h_{1}, h_{2}$ be any integer homology classes such that $h_{1} \cdot h_{2}=1$. Let $c_{1} \in h_{1}, c_{2} \in h_{2}$ be $g$-minimal periodic geodesics.

Let $f$ be a nonnegative $C^{\infty}$ function on $T^{2}$ satisfying the following conditions:

a) We have $f^{-1}(0)=c_{1}(\mathbb{R}) \cup c_{2}(\mathbb{R})$;

b) For $i=1,2$ there exists a unit tangent vector $v_{i}$ for the torus $\left(T^{2}, g\right)$ with base point in $c_{i}(\mathbb{R})$ which is normal to $c_{i}(\mathbb{R})$ and such that $(f \circ \gamma)^{\prime \prime}(0)>0$ for every curve $\gamma$ in $T^{2}$ with $\dot{\gamma}(0)=v_{1}$ or $\dot{\gamma}(0)=v_{2}$.

See figure 6, Let $\lambda>0$. We define the riemannian torus $\left(T^{2}, g_{\lambda}\right)$ by $g_{\lambda}=(1+\lambda f) g$. By condition $\left.a\right)$, the curves $c_{i}: \mathbb{R} \rightarrow T^{2}$ are the unique $g_{\lambda}$-minimal periodic geodesics in the classes $h_{i}, i=1,2$. We claim that

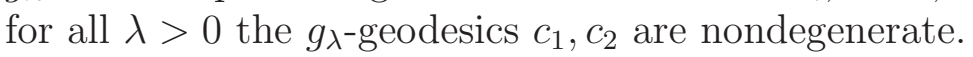

Assume the opposite, i. e., that for some $\lambda>0$ one of them, say $c_{1}$, is a degenerate $g_{\lambda}$-geodesic. Then there exists a nontrivial, periodic, normal $g_{\lambda^{-}}$Jacobi field $Y$ along $c_{1}$. Let $\exp _{\lambda}$ be the exponential map of $g_{\lambda}$. For $\tau \in \mathbb{R}$ denote by $\alpha_{\tau}(t)=\exp _{\lambda}(\tau Y(t))$ the normal variation of $c_{1}$ induced by $Y$. Let $L_{\lambda}$ denote the length with respect to $g_{\lambda}$; let $L$ be the length with respect to $g$. The formula for the second variation of arc-length 5 implies

$$
\left.\frac{d^{2}}{d \tau^{2}} L_{\lambda}\left(\alpha_{\tau}\right)\right|_{\tau=0}=0
$$

On the other hand, since the Jacobi field $Y$ has only isolated zeros, condition b) implies that for a nonempty open interval of values of $t$

\footnotetext{
${ }^{5}$ See, for instance, GKM], page 122, for the second variation of arclength formula.
} 


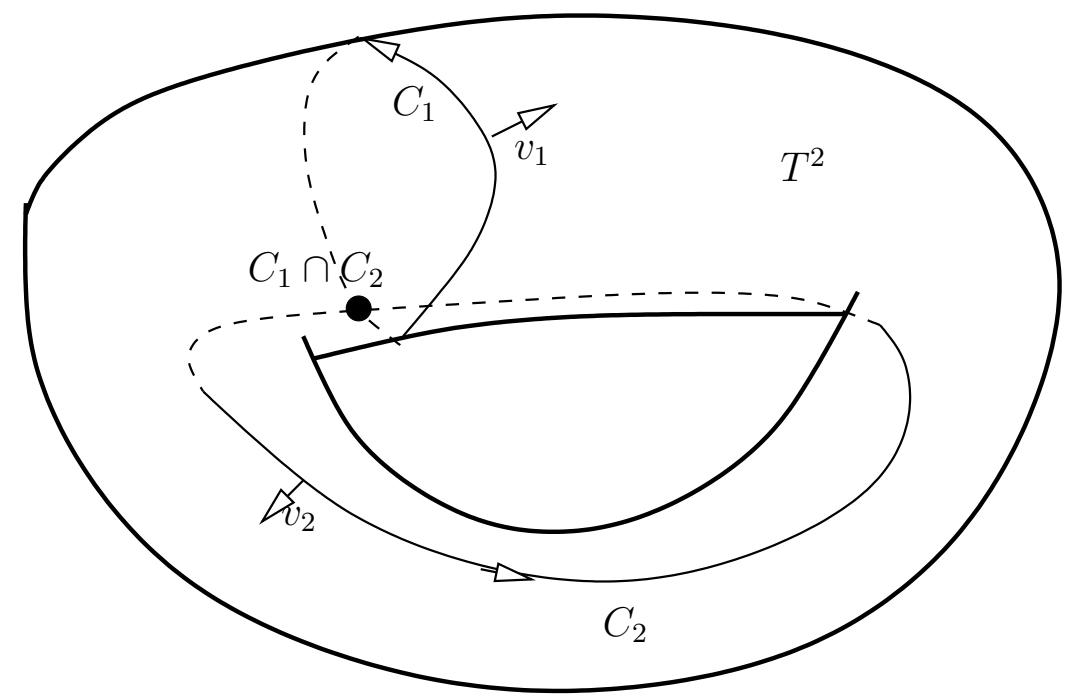

FiguRE 6. Illustration to the proof of Lemma 8.6.

we have

$$
\left.\frac{d^{2}}{d \tau^{2}} f\left(\exp _{\lambda}(\tau Y(t))\right)\right|_{\tau=0}>0
$$

Since $c_{1}$ is a $g$-minimal periodic geodesic, we have

$$
\left.\frac{d^{2}}{d \tau^{2}} L\left(\alpha_{\tau}\right)\right|_{\tau=0} \geq 0
$$

Differentiating the expression

$$
L_{\lambda}\left(\alpha_{\tau}\right)=\int_{0}^{L\left(c_{1}\right)}\left[\left(1+\lambda f\left(\exp _{\lambda}(\tau Y(t))\right)\right) g\left(\dot{\alpha}_{\tau}(t), \dot{\alpha}_{\tau}(t)\right)\right]^{\frac{1}{2}} d t
$$

and using equations (21) and (22), we obtain

$$
\left.\frac{d^{2}}{d \tau^{2}} L_{\lambda}\left(\alpha_{\tau}\right)\right|_{\tau=0}>0
$$

This contradicts equation (20), and hence proves our claim. Thus, $g_{\lambda} \in \mathcal{G}_{3}$ for all $\lambda>0$. By construction, $\left(T^{2}, g_{\lambda}\right)$ converges to $\left(T^{2}, g\right)$ as $\lambda \rightarrow 0$. Since $g$ is an arbitrary metric, this completes the proof.

Proof of Proposition 8.4. We recall that $\mathcal{G}_{1}$ (resp. $\mathcal{G}_{3}$ ) is the set of riemannian tori $\left(T^{2}, g\right)$ satisfying condition $\left(G_{1}\right)$ (resp. $\left(G_{3}\right)$ ). By Lemmas 8.5 and 8.6. $\mathcal{G}_{1}$ and $\mathcal{G}_{3}$ are $C^{2}$-open, hence the set $\mathcal{G}_{1} \cap \mathcal{G}_{3}$ is $C^{2}$-open as well. 
By Lemmas 8.5 and 8.6 , the sets $\mathcal{G}_{1}$ and $\mathcal{G}_{3}$ are $C^{\infty}$-dense. Since the $C^{\infty}$-topology is stronger than the $C^{2}$-topology, $\mathcal{G}_{1}$ and $\mathcal{G}_{3}$ are also $C^{\infty}$-open. The intersection of two open and dense sets is open and dense. Thus, the set $\mathcal{G}_{1} \cap \mathcal{G}_{3}$ is $C^{\infty}$-dense.

Theorem 8.3 and Proposition 8.4 immediately imply the following.

Corollary 8.7. The set of riemannian tori $\left(T^{2}, g\right)$ contains a $C^{2}$-open and $C^{\infty}$-dense subset of totally insecure tori.

Proof. Set $\mathcal{G}_{\text {tot }}=\mathcal{G}_{1} \cap \mathcal{G}_{3}$. By Proposition 8.4, $\mathcal{G}_{\text {tot }}$ is a $C^{2}$-open and $C^{\infty}$-dense subset of the set of riemannian tori. By Theorem 8.3 , for $g \in \mathcal{G}_{\text {tot }}$, every pair of points in $\left(T^{2}, g\right)$ is insecure.

\section{TOtAL INSECURITY FOR SURFACES OF GENUS GREATER THAN ONE}

In this section we will prove that on every closed riemannian surface $M$ of genus greater than one every point pair is insecure. Replacing $M$ by a two-sheeted covering space, if necessary, we can assume without loss of generality that $M$ is oriented. The basic idea is simple and has already been used to prove the total insecurity of admissible cylinders. See Theorem 6.2.

Besides the given metric $g$ on $M$, we consider a "background metric" $g_{0}$ of curvature -1 on $M$. Then the universal riemannian covering of $\left(M, g_{0}\right)$ can be identified with the open unit disc $D \subseteq \mathbb{C}$ with the standard hyperbolic metric; thus, we have a covering $\pi: D \rightarrow M$ such that $\tilde{g}_{0}=\pi^{*} g_{0}$ is the standard hyperbolic metric. We will say that the geodesics, distances, etc, with respect to $g_{0}$ or $\tilde{g}_{0}$ are the hyperbolic geodesics, distances, etc. Note that $\tilde{g}_{0}$ and the lifted metric $\tilde{g}=\pi^{*} g$ are lipshitz equivalent.

The proof of Theorem 9.7, which is the main result in this section, crucially uses fundamental facts due to M. Morse [Mo]. For convenience of the reader, we give their complete statements below.

Theorem 9.1 invokes the standard notion of the Hausdorff distance between subsets of a metric space. We do not specify the metric in the statement of the theorem because lipschitz equivalent metrics yield equivalent Hausdorff distances. By a geodesic segment we will mean the trace of a geodesic, when the endpoints are specified.

Theorem 9.1. ([Mo, Lemma 8). There is a positive constant $R$ such that the Hausdorff distance between any $\tilde{g}$-minimal geodesic segment 
and the hyperbolic geodesic segment with the same endpoints is less than $R$.

In view of Theorem 9.1 , every $\tilde{g}$-minimal geodesic $c: \mathbb{R} \rightarrow D$ is a uniformly bounded distance away from some hyperbolic geodesic. Therefore, there are distinct points $c(-\infty), c(\infty) \in \partial D$ such that we have $\lim _{t \rightarrow \pm \infty} c(t)=c( \pm \infty) .6$ The statement below directly follows from the fact that complete minimal geodesics intersect at most once.

Theorem 9.2. ([Mo, Theorem 4). Let $c_{1}: \mathbb{R} \rightarrow D$ and $c_{2}: \mathbb{R} \rightarrow$ $D$ be $\tilde{g}$-minimal geodesics. If $c_{2}(-\infty)$ and $c_{2}(\infty)$ belong to the same component of $\partial D \backslash\left\{c_{1}(-\infty), c_{1}(\infty)\right\}$ then the geodesics $c_{1}$ and $c_{2}$ do not intersect.

Our next statement directly follows from Theorem 9.2 .

Theorem 9.3. Let $c: \mathbb{R} / L \mathbb{Z} \rightarrow M$ be a periodic geodesic of minimal $g$-length in its free homotopy class. If this class contains a simple closed curve, then the mapping $c:[0, L) \rightarrow M$ is one-to-one.

Theorem 9.4. ([Mo, Theorem 11). Let $z, w \in \partial D$ be distinct points.

1. Suppose that there is more than one $\tilde{g}$-minimal geodesic $\tilde{c}: \mathbb{R} \rightarrow D$ with $\tilde{c}(-\infty)=z$ and $\tilde{c}(\infty)=w$. Then there exist two $\tilde{g}$-minimal geodesics $\tilde{c}_{ \pm}: \mathbb{R} \rightarrow D$ satisfying

$$
\tilde{c}_{ \pm}(-\infty)=z, \tilde{c}_{ \pm}(\infty)=w
$$

such that their traces bound a closed strip $S \subset D, S \simeq \mathbb{R} \times[0,1]$, containing every $\tilde{g}$-minimal geodesic with endpoints $z$ and $w$.

2. Suppose, in addition, that $z$ and $w$ are the endpoints of a lift of a closed curve in $M$. Let $\alpha$ be the free homotopy class of this curve. Then $\tilde{c}_{-}$and $\tilde{c}_{+}$project to periodic geodesics of minimal g-length in the class $\alpha$.

Let $\alpha$ be a nontrivial free homotopy class of closed curves in $M$ that contains a simple closed curve. We will construct a $\tilde{g}$-convex set $E \subseteq D$. In our proof of Theorem 9.7 the set $E$ will be a counterpart of the strip $S \subseteq \mathbb{R}^{2}$ in the proof of Theorem 6.2 on the insecurity of nonflat tori. There are two cases to consider.

Case (i): There is a unique periodic geodesic, say $c_{+}$, of minimal $g$ length in the homotopy class $\alpha$. We denote by $Z_{\alpha} \subset M$ the trace of $c_{+}$.

\footnotetext{
${ }^{6}$ The limits are with respect to the canonical topology on $\mathbb{C}$.
} 


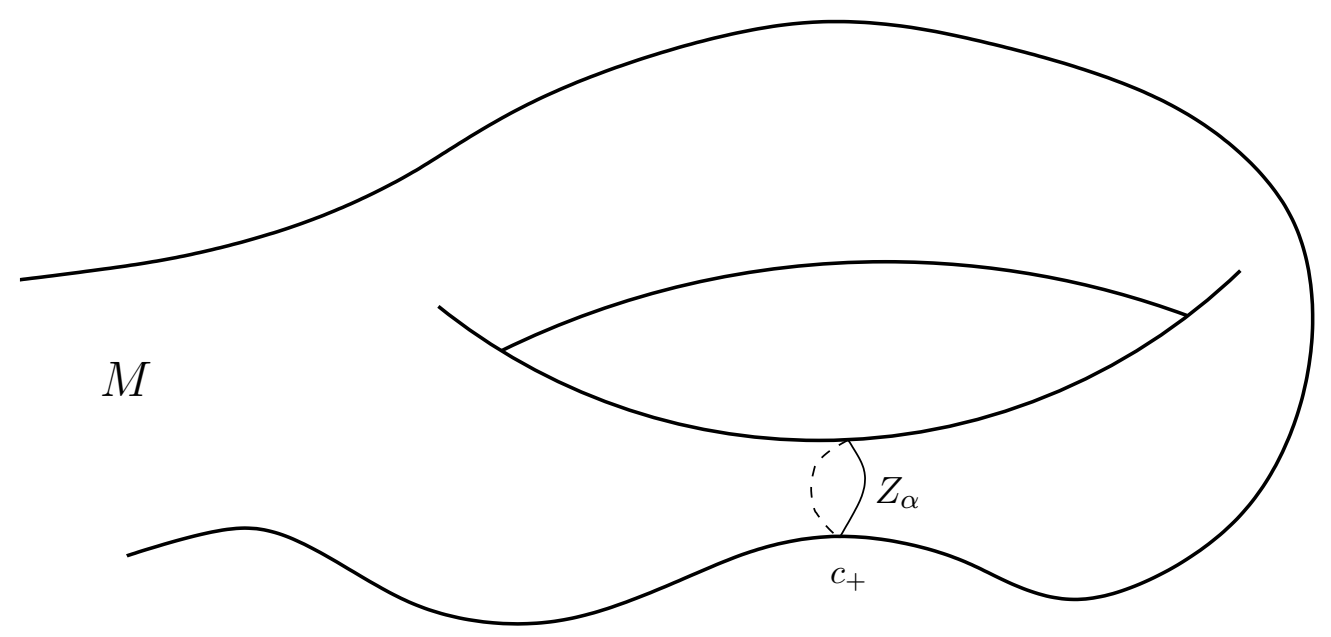

Figure 7. Case (i) : there is a unique closed geodesic of minimal length in the homotopy class $\alpha$.

Case (ii): There are at least two periodic geodesics of minimal $g$-length in the class $\alpha$. It follows from Theorem 9.3 and Theorem 9.4 that there exist two such geodesics bounding a closed cylinder $Z_{\alpha} \subset M$ that contains every periodic geodesic of minimal $g$-length in the class $\alpha$. We denote these geodesics by $c_{+}$and $c_{-}$, so that $Z_{\alpha}$ is to the left of $c_{-}$. Figure 7 and figure 8 illustrate the cases (i) and (ii) respectively. In particular, $Z_{\alpha} \simeq S^{1}$ in case (i) and $Z_{\alpha} \simeq S^{1} \times[0,1]$ in case (ii).

We choose a lift, say $\tilde{c}_{+}$, of $c_{+}$to $D$. Now we define $E$ to be the connected component of $D \backslash \pi^{-1}\left(Z_{\alpha}\right)$ whose boundary contains $\tilde{c}_{+}$and which lies to the left of $\tilde{c}_{+}$. Note that the latter condition is automatically satisfied in case (ii). See figure 9 and figure 10 for illustration.

Lemma 9.5. The set $E$ has the following properties:

a) If $x, y \in E$ then every $\tilde{g}$-minimal geodesic from $x$ to $y$ is contained in $E$.

b) Let $\tilde{\alpha}$ denote the covering transformation for $\pi: D \rightarrow M$ mapping $\tilde{c}_{+}(0)$ to $\tilde{c}_{+}\left(L\left(c_{+}\right)\right)$. Then $\tilde{\alpha}(E)=E$.

c) Suppose, in addition, that $\alpha$ is homologically nontrivial. Then $\pi(E)=M \backslash Z_{\alpha}$.

Proof. a) Assume the opposite. Let $c:[0, L] \rightarrow D$ be a $\tilde{g}$-minimal geodesic from $x$ to $y$ intersecting $\partial E$. Let $t_{0} \in(0, L)$ be the first time that $c$ intersects $\partial E$. Then there exists a lift $\tilde{c}: \mathbb{R} \rightarrow D$ of $c_{+}$or $c_{-}$ such that $c\left(t_{0}\right) \in \tilde{c}(\mathbb{R})$ and $c\left(\left[0, t_{0}\right)\right) \subset E$. 


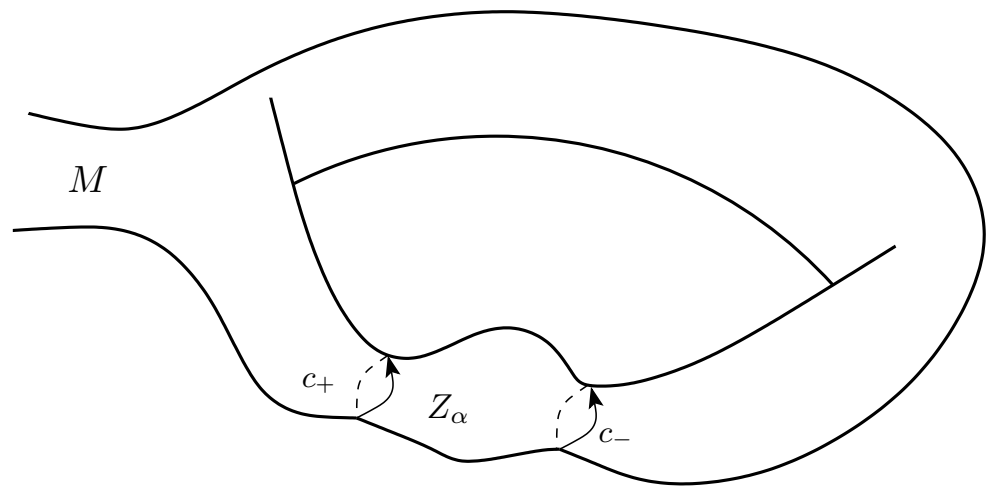

Figure 8. Case (ii) : there are at least two closed geodesics of minimal length in the homotopy class $\alpha$.

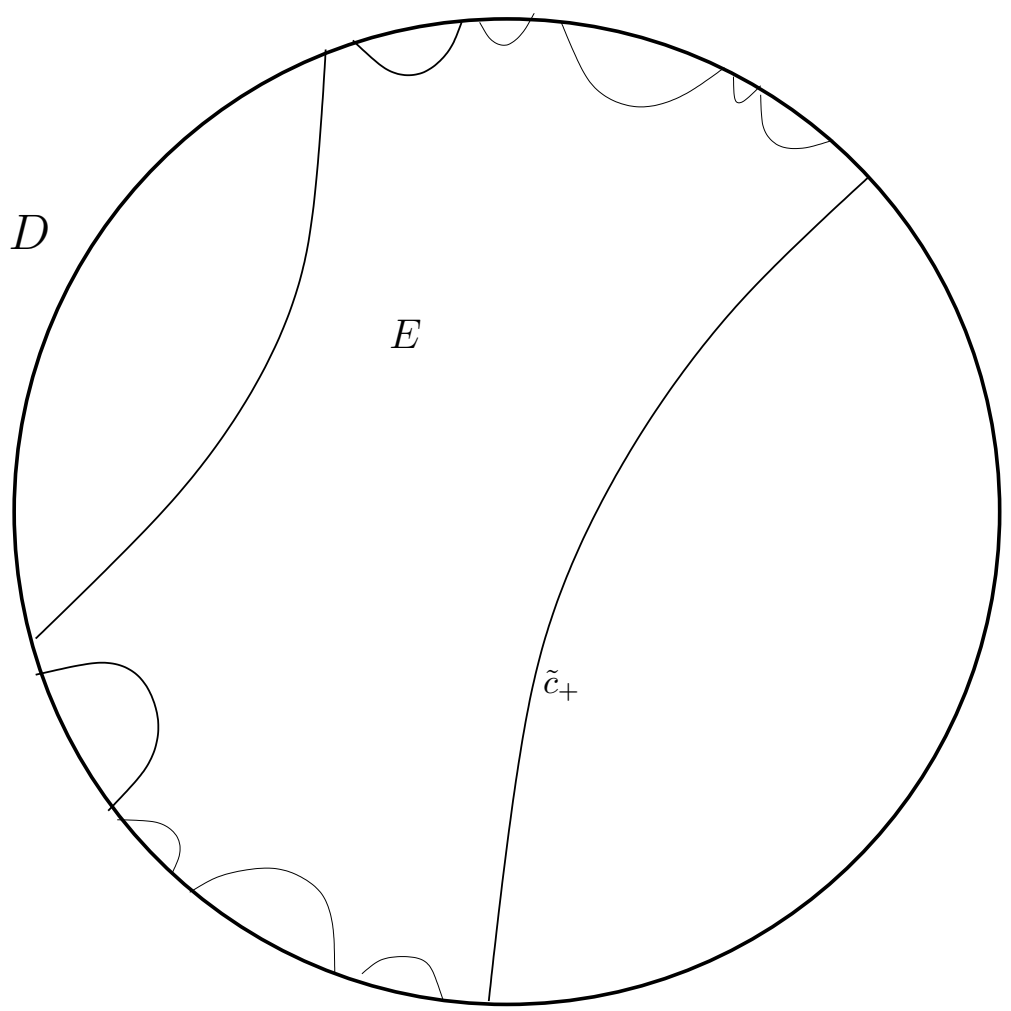

Figure 9. The region $E$ in the case when there is a unique closed geodesic of minimal length in the homotopy class $\alpha$. 


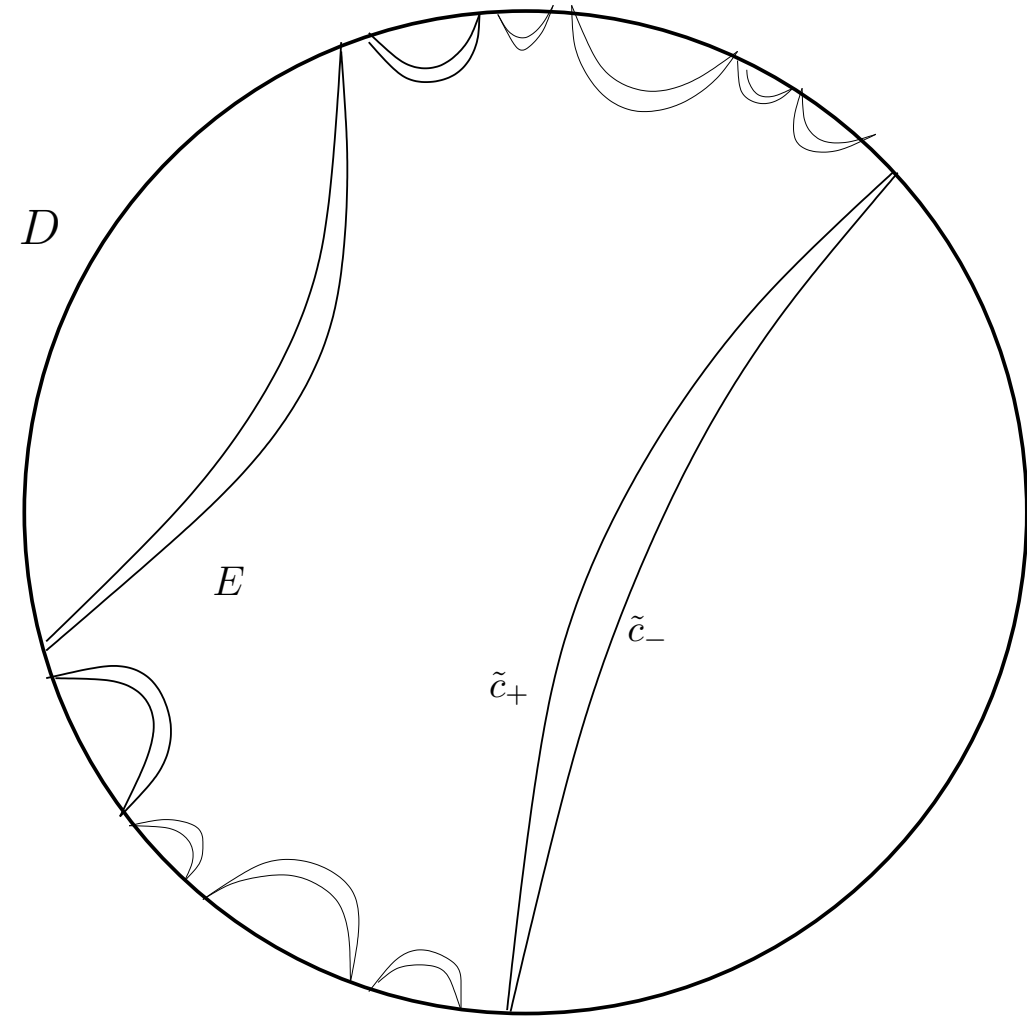

Figure 10. The region $E$ in the case when there are at least two closed geodesics of minimal length in the homotopy class $\alpha$.

Since $\tilde{c}(\mathbb{R})$ disconnects $D$ and since the geodesics $c$ and $\tilde{c}$ intersect transversely, there exists $t_{1} \in\left(t_{0}, L\right)$ such that $c\left(t_{1}\right) \in \tilde{c}(\mathbb{R})$. By Theorem 2.3, the geodesic $\tilde{c}$ is $\tilde{g}$-minimal. Hence, the $\tilde{g}$-minimal geodesic $c$ intersects $\tilde{c}$ at most once.

b) Since $\tilde{\alpha}$ is a deck transformation, it preserves any set of the form $\pi^{-1}(X), X \subset M$. In particular, $\tilde{\alpha}\left(\pi^{-1}\left(Z_{\alpha}\right)\right)=\pi^{-1}\left(Z_{\alpha}\right)$. Besides, by our choice of $\alpha$, we have $\tilde{\alpha}\left(\tilde{c}_{+}(\mathbb{R})\right)=\tilde{c}_{+}(\mathbb{R})$. Since $\tilde{\alpha}$ preserves the orientation, the claim follows.

c) Let $p \in M \backslash Z_{\alpha}$ be an arbitrary point. The homology class of $\alpha$ is nontrivial if and only if the set $M \backslash Z_{\alpha}$ is connected. Therefore, there is a regular $C^{1}$-path $\gamma:[0,1] \rightarrow M$ such that $\gamma(0)=c_{+}(0)$, $\gamma((0,1)) \subset M \backslash Z_{\alpha}, \gamma(1)=p$ and the pair $\left(\dot{c}_{+}(0), \dot{\gamma}(0)\right)$ is positively oriented. 
Let $\tilde{\gamma}:[0,1] \rightarrow D$ be the lift of $\gamma$ to $D$ starting at $\tilde{c}_{+}(0)$. Then $\tilde{\gamma}((0,1]) \subset E$, and hence $\tilde{\gamma}(1) \in E$. Since $\pi(\tilde{\gamma}(1))=p$, the claim follows.

In the following lemma we crucially use that the genus of $M$ is greater than one.

Lemma 9.6. For every pair $(p, q) \in M \times M$ there exists a free homotopy class $\alpha$ of closed curves in $M$ with the following properties:

a) The class $\alpha$ is homologically nontrivial and contains a simple closed curve;

b) We have $\{p, q\} \subset M \backslash Z_{\alpha}$.

Proof. Since the genus of $M$ is at least two, there exist free homotopy classes of closed curves in $M$, say $\alpha_{1}, \alpha_{2}, \alpha_{3}$, that satisfy condition a) and have pairwise disjoint representatives. Moreover, we can assume that the homology classes of $\alpha_{1}, \alpha_{2}, \alpha_{3}$ are pairwise linearly independent. See figure 11.

Let $Z_{\alpha_{1}}, Z_{\alpha_{2}}$ and $Z_{\alpha_{3}}$ be the corresponding subsets of $M$. See the discussion following Theorem 9.4. It follows from Theorem 9.2 that the sets $Z_{\alpha_{1}}, Z_{\alpha_{2}}$ and $Z_{\alpha_{3}}$ are pairwise disjoint. Therefore at least one of the classes $\alpha_{1}, \alpha_{2}, \alpha_{3}$ satisfies condition b).

We will now prove the main result of this section.

Theorem 9.7. Every closed riemannian surface $M$ of genus greater than one is totally insecure.

Proof. Let $(p, q) \in M \times M$ be an arbitrary pair of points. We choose a free homotopy class $\alpha$ according to Lemma 9.6. let $E \subset D$ be as in Lemma 9.5. By claim c) of Lemma 9.5, there exist points $x, y \in E$ such that $\pi(x)=p, \pi(y)=q$. Let $\tilde{\alpha}: D \rightarrow D$ be the covering transformation from Lemma $9.5 \mathrm{~b})$. For $n \in \mathbb{Z}$ we set $y_{n}=\tilde{\alpha}^{n}(y)$.

By claim b) of Lemma 9.5, $y_{n} \in E$ for all $n \in \mathbb{Z}$. For every positive integer $n$ let $\tilde{c}_{n}:\left[0, L_{n}\right] \rightarrow D$ be a $\tilde{g}$-minimal geodesic from $x$ to $y_{n}$. By claim a) of Lemma $\left[9.5\right.$, all curves $\tilde{c}_{n}\left(\left[0, L_{n}\right]\right)$ are contained in $E$. We will show that the projections $c_{n}=\pi \circ \tilde{c}_{n}:\left[0, L_{n}\right] \rightarrow M$ satisfy conditions a)-d) of Proposition 6.1, if we set $A=Z_{\alpha}$.

Let $\tilde{d}(\cdot, \cdot))$ be the distance in $D$ corresponding to the metric $\tilde{g}$. Since $\lim _{n \rightarrow \infty} \tilde{d}\left(x, y_{n}\right)=\infty$, we have $\lim L_{n}=\infty$, i. e. condition a) of Proposition 6.1 is satisfied. The curves $\tilde{c}_{n}\left(\left[0, L_{n}\right]\right)$ are contained in $E$ and, by claim c) of Lemma 9.5, $\pi(E) \subset M \backslash A$; hence condition b) holds. Since 
the geodesics $\tilde{c}_{n}$ are $\tilde{g}$-minimal, the geodesic segments $c_{n}\left(\left[0, L_{n}\right)\right)$ have no conjugate points, which verifies condition c).

Suppose now that condition d) of Proposition 6.1 is not fulfilled. Then there is $\varepsilon>0$, such that for all $k \in \mathbb{N}$ there is an integer $n_{k}>0$ and a number $t_{k} \in\left[k, L_{n_{k}}-k\right]$ such that

$$
\tilde{d}\left(\tilde{c}_{n_{k}}\left(t_{k}\right), \tilde{c}_{+}(\mathbb{R})\right) \geq \varepsilon .
$$

Observe that $\tilde{d}\left(\tilde{c}_{n}\left(L_{n}\right), \tilde{c}_{+}(\mathbb{R})\right)=\tilde{d}\left(y, \tilde{c}_{+}(\mathbb{R})\right)<\infty$. Theorem 9.1 and standard facts from hyperbolic geometry imply that there exists a constant $R$ such that for all $n \in \mathbb{N}$ and all $t \in\left[0, L_{n}\right]$ we have

$$
\tilde{d}\left(\tilde{c}_{n}(t), \tilde{c}_{+}(\mathbb{R})\right) \leq R .
$$

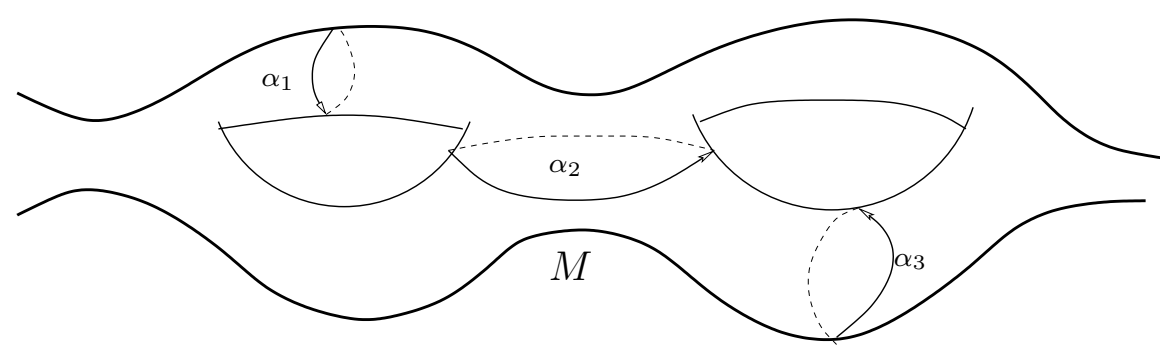

FigURE 11. Illustration to the proof of Lemma 9.6.

The group $\left\{\tilde{\alpha}^{n}: n \in \mathbb{Z}\right\}$ acts cocompactly on the set $\{z \in D$ : $\left.\tilde{d}\left(z, \tilde{c}_{+}(\mathbb{R})\right) \leq R\right\}$. Therefore, there is a sequence $m_{k}$ such that the sequence of geodesics

$$
t \rightarrow \tilde{\alpha}^{m_{k}} \circ \tilde{c}_{n_{k}}\left(t_{k}+t\right)
$$

converges to a $\tilde{g}$-minimal geodesic $c: \mathbb{R} \rightarrow \bar{E}$.

By equation (25), the geodesic $c$ satisfies $\tilde{d}\left(c(t), \tilde{c}_{+}(\mathbb{R})\right) \leq R$ for all $t \in \mathbb{R}$; hence

$$
\{c(\infty), c(-\infty)\}=\left\{\tilde{c}_{+}(\infty), \tilde{c}_{+}(-\infty)\right\} .
$$

Equation (26) and Theorem 9.4 imply that the trace of $c$ is either equal to the trace of $\tilde{c}_{+}$(in Case (i)) or is contained in the closed strip $S$ bounded by the traces of $\tilde{c}_{+}$and $\tilde{c}_{-}$(in Case (ii)). Since $c(\mathbb{R}) \subset \bar{E}$, we conclude that $c=\tilde{c}_{+}$, up to parameterization. On the other hand, since $c(0)$ is the limit point of the sequence $\tilde{\alpha}^{m_{k}} \circ \tilde{c}_{n_{k}}\left(t_{k}\right)$, by equation (24), we have $\left.\tilde{d}(c(0)), \tilde{c}_{+}(\mathbb{R})\right) \geq \varepsilon>0$, a contradiction.

We have shown that condition d) of Proposition 6.1 also holds. Thus, Proposition 6.1 applies and establishes the claim. 


\section{REFERENCES}

[Ba1] V. Bangert, Mather sets for twist maps and geodesics on tori, Dynamics Reported 1 (1988), 1 - 56.

[Ba2] V. Bangert, Geodesic rays, Busemann functions and monotone twist maps, Calc. Var. 2 (1994), 49 - 63.

[Ba3] V. Bangert, Hypersufaces without self-intersections in the torus, pp. $55-71$ in Twist Mappings and their Applications, IMA Proceedings, vol. 44, SpringerVerlag, Berlin, 1992.

[Ba4] V. Bangert, On riemannian two-tori with a pole, manuscript in preparation (2009).

[BaG08] V. Bangert and E. Gutkin, Secure two-dimensional tori are flat, preprint arXiv:0806.3572 (2008).

[BG08] K. Burns and E. Gutkin, Growth of the number of geodesics between points and insecurity for riemannian manifolds, Discr. \& Cont. Dyn. Sys. A 21 (2008), $403-413$.

[Bu] H. Busemann, The Geometry of Geodesics, Academic Press, New York, 1955.

[GKM] D. Gromoll, W. Klingenberg, W. Meyer, Riemannsche Geometrie im Grossen, Lecture Notes in Mathematics 55, Springer-Verlag, Berlin, 1968.

[Gut05] E. Gutkin, Blocking of billiard orbits and security for polygons and flat surfaces, GAFA: Geom. \& Funct. Anal. 15 (2005), 83 - 105.

[Gut09] E. Gutkin, Topological entropy and blocking cost for geodesics in Riemannian manifolds, Geom. Dedicata 138 (2009), 13 - 23.

[GS06] E. Gutkin and V. Schroeder, Connecting geodesics and security of configurations in compact locally symmetric spaces, Geom. Dedicata 118 (2006), 185 $-208$.

[He] G.A. Hedlund, Geodesics on a two-dimensional Riemannian manifold with periodic coefficients, Ann. Math. 33 (1932), 719 - 739.

[Her] P. Herreros, Blocking: new examples and properties of products, Erg. Theory \& Dyn. Sys. 29 (2009), 569 - 578.

[Hi] M.W. Hirsch, Differential Topology, Springer-Verlag, New York, 1976.

[Ho] E. Hopf, Closed surfaces without conjugate points, Proc. Nat. Acad. Sci. USA 34 (1948), $47-51$.

[In] N. Innami, Families of geodesics which distinguish flat tori, Math. J. Okayama Univ. 28 (1986), $207-217$.

[Kl] W. Klingenberg, A course in differential geometry, Springer-Verlag, New York, 1978

[LS07] J.-F. Lafont and B. Schmidt, Blocking light in compact Riemannian manifolds, Geometry \& Topology 11 (2007), 867 - 887.

[Mat] J.N. Mather, Action minimizing invariant measures for positive definite lagrangian systems, Math. Z. 207 (1991), 169 - 207. 
[Mo] M. Morse, A fundamental class of geodesics on any closed surface of genus greater than one, Trans. Amer. Math. Soc. 26 (1924), 25 - 60.

[Sch] S. Schwartzman, Asymptotic cycles, Ann. Math. 66 (1957), 270 - 284.

[So] B. Solomon, On foliations of $\mathbb{R}^{n+1}$ by minimal hypersurfaces, Comment. Math. Helv. 61 (1986), $67-83$.

[We] A. Weil, On systems of curves on a ring-shaped surface, J. Indian Math. Soc. 19 (1931), $109-114$.

[WKH] Wing Kai Ho, On Blocking Numbers of Surfaces, preprint arXiv:0807.2934 (2008).

Mathematisches Institut, Albert-Ludwigs-Universität, Eckerstrasse 1, 79104 Freiburg im Breisgau, Germany

E-mail address: bangert@email.mathematik.uni-freiburg.de

Nicolaus Copernicus University (UMK), and Mathematics Institute of the Polish Academy of Sciences (IMPAN), Chopina 12/18, 87-100 Torun, Poland

E-mail address: gutkin@mat.umk.pl, gutkin@impan.pl 